CERN-TH.6920/93

UWThPh-1993-22

\title{
Non-Leptonic Kaon Decays and the Chiral Anomaly*
}

\author{
G. Ecker, H. Neufeld \\ Institut für Theoretische Physik \\ Universität Wien \\ Boltzmanngasse 5, A-1090 Wien \\ and
}

A. Pich

CERN

CH-1211 Geneva 23

\begin{abstract}
An investigation is performed of all non-leptonic kaon decays sensitive to the chiral anomaly. Within the framework of chiral perturbation theory, there are two classes of anomalous amplitudes at $O\left(p^{4}\right)$ : reducible and direct contributions. Only radiative transitions are affected by the anomaly. The phenomenology of the decays $K_{L} \rightarrow \pi^{+} \pi^{-} \gamma$ and $K^{+} \rightarrow \pi^{+} \pi^{0} \gamma$ is studied in detail. Including the dominant contributions of $O\left(p^{6}\right)$, the experimentally observed dependence of the direct emission amplitude for $K_{L} \rightarrow \pi^{+} \pi^{-} \gamma$ on the photon energy can be understood. A survey is made of the rare "anomalous" decays $K \rightarrow \pi \pi \gamma \gamma$ and $K \rightarrow 3 \pi \gamma(\gamma)$, including some numerical estimates.

* Work supported in part by Fonds zur Förderung der wiss. Forschung (Austria), Project Nos. P08485-TEC, P09505-PHY (EURODAPHNE Collaboration) and by CICYT (Spain), grant No. AEN90-0040
\end{abstract}

CERN-TH.6920/93

June 1993 


\section{Introduction}

The standard model is a chiral quantum field theory. The chiral structure is responsible for the existence of the chiral anomaly $[1,2]$ whose theoretical origin and mathematical properties are well understood. On the other hand, experimental tests of this important ingredient of modern particle physics are relatively rare.

The chiral anomaly manifests itself most directly in the low-energy interactions of the pseudoscalar mesons (some of the phenomenological aspects can be found in the recent reviews $[3,4,5])$. The appropriate framework to study these effects is chiral perturbation theory (CHPT) [6, 7, 8]. For the strong, electromagnetic and semileptonic weak interactions, all anomalous Green functions can be obtained from the Wess-Zumino-Witten (WZW) functional [9]. In contrast to most other aspects of the standard model in the world of hadrons, the translation from the fundamental level to the effective chiral level is unambiguous and free from hadronization problems.

The chiral anomaly also appears in the non-leptonic weak interactions. The purpose of this paper is to give a systematic account of all non-leptonic $K$ decays where the anomaly contributes at leading order, $O\left(p^{4}\right)$. As already shown in previous work $[10,11]$, only radiative $K$ decays are sensitive to the chiral anomaly in the non-leptonic sector. There are two different manifestations of the anomaly: the reducible amplitudes [10], which can again be derived directly from the WZW functional, and direct contributions $[11,12]$, which are subject to some theoretical uncertainties. We shall present a selfcontained and systematic discussion of those contributions. As a special application, the two most frequent "anomalous" decays $K_{L} \rightarrow \pi^{+} \pi^{-} \gamma$ and $K^{+} \rightarrow \pi^{+} \pi^{0} \gamma$ will be analysed in detail, including the dominant effects of $O\left(p^{6}\right)$. In both cases, the direct emission amplitudes are dominated by the anomaly. A careful treatment of $O\left(p^{6}\right)$ effects is necessary to understand the experimentally observed dependence of the direct emission amplitude on the photon energy for the decay $K_{L} \rightarrow \pi^{+} \pi^{-} \gamma$.

In Sect. 2, we summarize the chiral realization of the $\Delta S=1$ non-leptonic weak interactions in the meson sector. The relevant terms of the strong and weak Lagrangians of $O\left(p^{4}\right)$ are listed. The distinction between reducible and direct anomalous amplitudes is explained in Sect. 3 . The direct weak anomaly functional is related to the general weak Lagrangian of $O\left(p^{4}\right)$. A list of all 
non-leptonic $K$ decays with local anomalous amplitudes of $O\left(p^{4}\right)$ is given. General features of the decays $K \rightarrow \pi \pi \gamma$ are put together in the following section. A general theorem on the structure of the lowest-order amplitudes for those decays is formulated and discussed. The dominant effects of $O\left(p^{6}\right)$ are expected to be due to vector meson exchange. The factorization model is proposed to estimate the direct weak terms related to $\mathrm{V}$ exchange.

We turn to the phenomenology of $K \rightarrow \pi \pi \gamma$ decays in Sect. 5. For completeness, we include a brief review of the theoretical status of the decays $K_{L, S} \rightarrow \pi^{0} \pi^{0} \gamma$ and $K_{S} \rightarrow \pi^{+} \pi^{-} \gamma$ even though they are not subject to anomalous contributions. Our main emphasis, however, is on the anomalous decays $K_{L} \rightarrow \pi^{+} \pi^{-} \gamma$ and $K^{+} \rightarrow \pi^{+} \pi^{0} \gamma$. In both cases, the bremsstrahlung amplitudes are suppressed as are the one-loop amplitudes. A careful analysis of the magnetic amplitudes of $O\left(p^{6}\right)$ is made since experiments are already sensitive to those subleading contributions. We suggest an interpretation of the measured slope parameter in the $K_{L}$ decay $[13,14,15]$ and compare with previous work by other authors. In Sect. 6, on overview of the remaining non-leptonic $K$ decays sensitive to the anomaly is given. In addition to some comments on the general structure of those decays, numerical results are presented for two typical transitions, $K^{+} \rightarrow \pi^{+} \pi^{0} \gamma \gamma$ and $K_{L} \rightarrow \pi^{+} \pi^{-} \pi^{0} \gamma$. Our findings are summarized in Sect. 7. Two Appendices contain a proof of the bremsstrahlung theorem of Sect. 4 and the definition of loop functions encountered in Sect. 5.

\section{CHPT for non-leptonic weak interactions}

At low energies $\left(E \ll M_{W}\right)$, the $\Delta S=1$ non-leptonic weak interactions are described by an effective Hamiltonian [16]

$$
\mathcal{H}_{\mathrm{eff}}^{\Delta S=1}=\frac{G_{F}}{\sqrt{2}} V_{u d} V_{u s}^{*} \sum_{i} C_{i} Q_{i}+\text { h.c. }
$$

in terms of Wilson coefficients $C_{i}$ and local four-quark operators $Q_{i}$. The effective chiral Lagrangian for (2.1) to lowest order in the chiral expansion can be written as ( $F$ is the pion decay constant in the chiral limit, $F \simeq F_{\pi}=$ 
$93.2 \mathrm{MeV})$ :

$$
\begin{gathered}
\mathcal{L}_{2}^{\Delta S=1}=G_{8} F^{4}\left\langle\lambda L_{\mu} L^{\mu}\right\rangle+G_{27} F^{4}\left(L_{\mu 23} L_{11}^{\mu}+\frac{2}{3} L_{\mu 21} L_{13}^{\mu}\right)+\text { h.c. } \\
\lambda=\frac{1}{2}\left(\lambda_{6}-i \lambda_{7}\right), \quad L_{\mu}=i U^{\dagger} D_{\mu} U, \quad\langle A\rangle=\operatorname{tr} A .
\end{gathered}
$$

The matrix field $U(\varphi)$ incorporating the eight pseudoscalar Goldstone boson fields transforms linearly under the chiral group $S U(3)_{L} \times S U(3)_{R}$. The covariant derivative

$$
D_{\mu} U=\partial_{\mu} U-i r_{\mu} U+i U \ell_{\mu}
$$

with $3 \times 3$ Hermitian matrix fields $\ell_{\mu}, r_{\mu}$ contains in particular the photon field:

$$
\begin{aligned}
r_{\mu} & =v_{\mu}+a_{\mu}=e Q A_{\mu}+\ldots \\
\ell_{\mu} & =v_{\mu}-a_{\mu}=e Q A_{\mu}+\ldots \\
Q & =\frac{1}{3} \operatorname{diag}(2,-1,-1)
\end{aligned}
$$

where $Q$ is the quark charge matrix.

The coupling constants $G_{8}, G_{27}$ in (2.2) measure the strength of the two parts in the effective Hamiltonian (2.1) transforming as $\left(8_{L}, 1_{R}\right)$ and $\left(27_{L}, 1_{R}\right)$, respectively, under chiral rotations. Neglecting the small $\Delta I=1 / 2$ part of the 27-plet, the Lagrangian (2.2) produces the tree-level amplitudes

$$
\begin{aligned}
A\left(K_{1}^{0} \rightarrow \pi^{+} \pi^{-}\right) & =2 i F\left(G_{8}+G_{27}^{(3 / 2)}\right)\left(M_{\pi}^{2}-M_{K}^{2}\right) \\
A\left(K^{+} \rightarrow \pi^{+} \pi^{0}\right) & =3 i F G_{27}^{(3 / 2)}\left(M_{\pi}^{2}-M_{K}^{2}\right) \\
G_{27}^{(3 / 2)} & =\frac{5}{9} G_{27} .
\end{aligned}
$$

Up to radiative and higher-order chiral corrections $[17,18]$, the ratio

$$
\frac{G_{27}^{(3 / 2)}}{G_{8}}=\frac{1}{32}
$$

is small (and positive), expressing the $\Delta I=1 / 2$ rule in $K \rightarrow 2 \pi$ decays, and $\left|G_{8}\right| \simeq 9 \cdot 10^{-6} \mathrm{GeV}^{-2}$. 
At next-to-leading order in CHPT, the chiral Lagrangian $\mathcal{L}_{4}^{\Delta S=1}$ is already quite involved [19]. We shall only need the octet Lagrangian of $O\left(p^{4}\right)$. Employing the operator basis of Ref. [20], we write

$$
\mathcal{L}_{4}^{\Delta S=1}=G_{8} F^{2} \sum_{i} N_{i} W_{i}+\text { h.c. }
$$

with dimensionless coupling constants $N_{i}$ and octet operators $W_{i}$. Referring to Ref. [20] for the complete Lagrangian, we list here only those terms that will be needed in the following. To facilitate the use of this effective Lagrangian, we write down the relevant operators in two different representations commonly used in CHPT:

$$
\begin{aligned}
& W_{14}=i\left\langle\Delta\left\{f_{+}^{\mu \nu}, u_{\mu} u_{\nu}\right\}\right\rangle=i\left\langle\lambda\left\{F_{L}^{\mu \nu}+U^{\dagger} F_{R}^{\mu \nu} U, L_{\mu} L_{\nu}\right\}\right\rangle \\
& W_{15}=i\left\langle\Delta u_{\mu} f_{+}^{\mu \nu} u_{\nu}\right\rangle=i\left\langle\lambda L_{\mu}\left(F_{L}^{\mu \nu}+U^{\dagger} F_{R}^{\mu \nu} U\right) L_{\nu}\right\rangle \\
& W_{16}=i\left\langle\Delta\left\{f_{-}^{\mu \nu}, u_{\mu} u_{\nu}\right\}\right\rangle=i\left\langle\lambda\left\{F_{L}^{\mu \nu}-U^{\dagger} F_{R}^{\mu \nu} U, L_{\mu} L_{\nu}\right\}\right\rangle \\
& W_{17}= i\left\langle\Delta u_{\mu} f_{-}^{\mu \nu} u_{\nu}\right\rangle=i\left\langle\lambda L_{\mu}\left(F_{L}^{\mu \nu}-U^{\dagger} F_{R}^{\mu \nu} U\right) L_{\nu}\right\rangle \\
& W_{18}=\left\langle\Delta\left(f_{+\mu \nu} f_{+}^{\mu \nu}-f_{-\mu \nu} f_{-}^{\mu \nu}\right)\right\rangle=2\left\langle\lambda\left(F_{L}^{\mu \nu} U^{\dagger} F_{R \mu \nu} U+U^{\dagger} F_{R \mu \nu} U F_{L}^{\mu \nu}(2) .8\right)\right. \\
& W_{28}=i \varepsilon_{\mu \nu \rho \sigma}\left\langle\Delta u^{\mu}\right\rangle\left\langle u^{\nu} u^{\rho} u^{\sigma}\right\rangle=i \varepsilon_{\mu \nu \rho \sigma}\left\langle\lambda L^{\mu}\right\rangle\left\langle L^{\nu} L^{\rho} L^{\sigma}\right\rangle \\
& W_{29}=\left\langle\Delta\left[\widetilde{f}_{+}^{\mu \nu}-\widetilde{f}_{-}^{\mu \nu}, u_{\mu} u_{\nu}\right]\right\rangle=2\left\langle\lambda\left[U^{\dagger} \widetilde{F}_{R}^{\mu \nu} U, L_{\mu} L_{\nu}\right]\right\rangle \\
& W_{30}=\left\langle\Delta u_{\mu}\right\rangle\left\langle\widetilde{f}_{+}^{\mu \nu} u_{\nu}\right\rangle=\left\langle\lambda L_{\mu}\right\rangle\left\langle\left(\widetilde{F}_{L}^{\mu \nu}+U^{\dagger} \widetilde{F}_{R}^{\mu \nu} U\right) L_{\nu}\right\rangle \\
&\left.W_{31}=\left\langle\Delta u_{\mu}\right\rangle\left\langle\widetilde{f}_{-}^{\mu \nu} u_{\nu}\right\rangle=\left\langle\lambda L_{\mu}\right\rangle\left\langle\widetilde{F}_{L}^{\mu \nu}-U^{\dagger} \widetilde{F}_{R}^{\mu \nu} U\right) L_{\nu}\right\rangle \\
& U=u^{2}, \\
& U_{\mu}=u \lambda u^{\dagger}, \quad u_{\mu}=i u^{\dagger} D_{\mu} U u^{\dagger}=u L_{\mu} u^{\dagger}, \quad f_{ \pm}^{\mu \nu}=u F_{L}^{\mu \nu} u^{\dagger} \pm u^{\dagger} F_{R}^{\mu \nu} u .
\end{aligned}
$$

$F_{L}^{\mu \nu}, F_{R}^{\mu \nu}$ are the field strength tensors associated to the external gauge fields $\ell_{\mu}, r_{\mu}[8]$ and $\widetilde{F}_{L, R \mu \nu}=\varepsilon_{\mu \nu \rho \sigma} F_{L, R}^{\rho \sigma}$ are their duals.

To calculate non-leptonic weak amplitudes, we also need the chiral Lagrangian for the strong, electromagnetic and semileptonic weak interactions. At lowest order, it is given by

$$
\mathcal{L}_{2}=\frac{F^{2}}{4}\left\langle D_{\mu} U D^{\mu} U^{\dagger}+2 B_{0} \mathcal{M}\left(U+U^{\dagger}\right)\right\rangle
$$

in the notation of [8], where $\mathcal{M}$ is the diagonal quark mass matrix and $B_{0}$ is related to the quark condensate.

Of the strong chiral Lagrangian of $O\left(p^{4}\right)$ [8] we shall encounter only one term,

$$
\mathcal{L}_{4}=-i L_{9}\left\langle F_{L}^{\mu \nu} D_{\mu} U^{\dagger} D_{\nu} U+F_{R}^{\mu \nu} D_{\mu} U D_{\nu} U^{\dagger}\right\rangle+\ldots
$$


Like many of the weak couplings $N_{i}$ in (2.7), the measurable (renormalized) coupling constant $L_{9}^{r}(\mu)$ is scale-dependent due to the divergences of the oneloop functional [8]. In Sect. 5 we shall use the standard value [8] $L_{9}^{r}\left(M_{\rho}\right) \simeq$ $6.9 \cdot 10^{-3}$.

Finally, a crucial ingredient of our analysis is the chiral anomaly, which also enters the effective description at $O\left(p^{4}\right)$. It will be dealt with in the following section.

\section{The chiral anomaly in the non-leptonic weak sector}

The contributions of the chiral anomaly to strong, electromagnetic and semileptonic weak amplitudes can be expressed in terms of the Wess-ZuminoWitten (WZW) functional [9] $S[U, \ell, r]_{W Z W}$. If the vector currents are to be conserved, it has the following explicit form :

$$
\begin{aligned}
& S[U, \ell, r]_{W Z W}=-\frac{i N_{C}}{240 \pi^{2}} \int d \sigma^{i j k l m}\left\langle\Sigma_{i}^{L} \Sigma_{j}^{L} \Sigma_{k}^{L} \Sigma_{l}^{L} \Sigma_{m}^{L}\right\rangle \\
&-\frac{i N_{C}}{48 \pi^{2}} \int d^{4} x \varepsilon_{\mu \nu \alpha \beta}\left(W(U, \ell, r)^{\mu \nu \alpha \beta}-W(\mathbf{1}, \ell, r)^{\mu \nu \alpha \beta}\right) \\
& W(U, \ell, r)_{\mu \nu \alpha \beta}=\left\langle U \ell_{\mu} \ell_{\nu} \ell_{\alpha} U^{\dagger} r_{\beta}+\frac{1}{4} U \ell_{\mu} U^{\dagger} r_{\nu} U \ell_{\alpha} U^{\dagger} r_{\beta}+i U \partial_{\mu} \ell_{\nu} \ell_{\alpha} U^{\dagger} r_{\beta}\right. \\
&+i \partial_{\mu} r_{\nu} U \ell_{\alpha} U^{\dagger} r_{\beta}-i \Sigma_{\mu}^{L} \ell_{\nu} U^{\dagger} r_{\alpha} U \ell_{\beta}+\Sigma_{\mu}^{L} U^{\dagger} \partial_{\nu} r_{\alpha} U \ell_{\beta} \\
&-\Sigma_{\mu}^{L} \Sigma_{\nu}^{L} U^{\dagger} r_{\alpha} U \ell_{\beta}+\Sigma_{\mu}^{L} \ell_{\nu} \partial_{\alpha} \ell_{\beta}+\Sigma_{\mu}^{L} \partial_{\nu} \ell_{\alpha} \ell_{\beta} \\
&\left.-i \Sigma_{\mu}^{L} \ell_{\nu} \ell_{\alpha} \ell_{\beta}+\frac{1}{2} \Sigma_{\mu}^{L} \ell_{\nu} \Sigma_{\alpha}^{L} \ell_{\beta}-i \Sigma_{\mu}^{L} \Sigma_{\nu}^{L} \Sigma_{\alpha}^{L} \ell_{\beta}\right\rangle \\
&-(L \leftrightarrow R) \\
& \Sigma_{\mu}^{L}=U^{\dagger} \partial_{\mu} U \quad \Sigma_{\mu}^{R}=U \partial_{\mu} U^{\dagger} \\
& N_{C}=3 \quad \varepsilon_{0123}=1
\end{aligned}
$$

where $(L \leftrightarrow R)$ stands for the interchange

$$
U \leftrightarrow U^{\dagger}, \quad \ell_{\mu} \leftrightarrow r_{\mu}, \quad \Sigma_{\mu}^{L} \leftrightarrow \Sigma_{\mu}^{R} .
$$


The functional $S[U, \ell, r]_{W Z W}$ conserves parity and it reproduces the anomaly under chiral transformations in Bardeen's form [2]. The integration in the first term of Eq. (3.1) is over a five-dimensional manifold whose boundary is four-dimensional Minkowski space. Since the integrand is a surface term, both the first and the second term of $S_{W Z W}$ are $O\left(p^{4}\right)$, according to the usual chiral counting rules.

The chiral anomaly also contributes to non-leptonic weak amplitudes starting at $O\left(p^{4}\right)$. We may distinguish between two different manifestations of the anomaly.

\section{i. Reducible anomalous amplitudes}

These amplitudes arise from the contraction of meson lines between a weak $\Delta S=1$ Green function and the WZW functional. At $O\left(p^{4}\right)$, there can only be one such contraction and the weak vertex must be due to the lowest-order non-leptonic Lagrangian $\mathcal{L}_{2}^{\Delta S=1}$ in Eq. (2.2). The corresponding diagrams are of the type shown in Fig. ??.

Since $\mathcal{L}_{2}^{\Delta S=1}$ contains bilinear terms in the meson fields, the so-called pole contributions to anomalous non-leptonic amplitudes can be given in closed form by a simultaneous diagonalization [21] of the kinetic parts of the Lagrangians $\mathcal{L}_{2}$ and $\mathcal{L}_{2}^{\Delta S=1}$. The corresponding local Lagrangian (octet part only) is $[10]$ :

$\mathcal{L}_{\text {an }}^{\Delta S=1}=-\frac{i e G_{8}}{8 \pi^{2} F} \widetilde{F}^{\mu \nu} \partial_{\mu} \pi^{0} K^{+} \stackrel{\leftrightarrow}{D_{\nu}} \pi^{-}+\frac{\alpha G_{8}}{6 \pi F} \widetilde{F}^{\mu \nu} F_{\mu \nu}\left(K^{+} \pi^{-} \pi^{0}-\frac{1}{\sqrt{2}} K^{0} \pi^{+} \pi^{-}\right)+$h.c.

Here $F_{\mu \nu}=\partial_{\mu} A_{\nu}-\partial_{\nu} A_{\mu}$ is the electromagnetic field strength tensor, $\widetilde{F}_{\mu \nu}=\varepsilon_{\mu \nu \rho \sigma} F^{\rho \sigma}$ its dual and $D_{\mu} \varphi^{ \pm}=\left(\partial_{\mu} \mp i e A_{\mu}\right) \varphi^{ \pm}$denotes the covariant derivative with respect to electromagnetism. In the limit of CP conservation, the anomalous Lagrangian (3.3) contributes only to the decays

$$
K^{+} \rightarrow \pi^{+} \pi^{0} \gamma, \pi^{+} \pi^{0} \gamma \gamma \quad \text { and } \quad K_{L} \rightarrow \pi^{+} \pi^{-} \gamma \gamma
$$

with real or virtual photons.

There are of course other reducible anomalous amplitudes corresponding to the diagram in Fig. ??. A generic example is provided by a non-leptonic 
Green function where an external $\pi^{0}$ or $\eta$ makes an anomalous transition to two photons. Such transitions are the dominant $O\left(p^{4}\right)$ contributions to the decays $K_{S} \rightarrow \pi^{0} \gamma \gamma[22]$ and $K_{L} \rightarrow \pi^{0} \pi^{0} \gamma \gamma[23,24]$. All reducible anomalous amplitudes of $O\left(p^{4}\right)$ are proportional to $G_{8}$ in the octet limit. No other unknown parameters are involved.

\section{ii. Direct weak anomaly functional}

The second manifestation of the anomaly in non-leptonic weak amplitudes arises diagrammatically from the contraction of the $W$ boson field between a strong Green function on one side and the WZW functional on the other side. However, such diagrams cannot be taken literally at a typical hadronic scale, because of the presence of strongly interacting fields on both sides of the $W$. Instead, one must as in Sect. 2 first integrate out the $W$ together with the heavy quark fields. The operators appearing in the operator product expansion must then be realized at the bosonic level in the presence of the anomaly.

Following the methods of Ref. [25], the bosonization of four-quark operators in the odd-intrinsic parity sector was investigated in Ref. [11]. As in the even-intrinsic parity sector, the bosonized four-quark operators contain factorizable (leading in $1 / N_{C}$, where $N_{C}$ is the number of colours) and non-factorizable parts (non-leading in $1 / N_{C}$ ).

Due to the non-renormalization theorem [26] of the chiral anomaly, the factorizable contribution of $O\left(p^{4}\right)$ can be calculated exactly [11]. The bosonized form of a $(V-A) \times(V-A)$ four-quark operator in the anomalous sector is [factorizable contribution of $O\left(p^{4}\right)$ ]:

$$
\overline{q_{l L}} \gamma^{\mu} q_{k L} \overline{q_{j L}} \gamma_{\mu} q_{i L} \leftrightarrow \frac{\delta S_{W Z W}}{\delta \ell_{l k}^{\mu}} \frac{\delta S_{2}}{\delta \ell_{\mu, j i}}+(l k \leftrightarrow j i)
$$

where

$$
\begin{aligned}
\frac{\delta S_{2}}{\delta \ell_{\mu, j i}} & =-\frac{F^{2}}{2}\left(L^{\mu}\right)_{i j} \\
L^{\mu} & =i U^{\dagger} D^{\mu} U
\end{aligned}
$$


is the left-chiral current of lowest order $p$ corresponding to the chiral Lagrangian (2.9). The anomalous current [of $\left.O\left(p^{3}\right)\right]$ has the following form

$$
\begin{aligned}
\frac{\delta S_{W Z W}}{\delta \ell_{\mu, j i}} & =\frac{1}{16 \pi^{2}} \varepsilon^{\mu \nu \alpha \beta} J_{\nu \alpha \beta, i j}^{a n} \\
J_{\nu \alpha \beta}^{a n} & =i L_{\nu} L_{\alpha} L_{\beta}+\left\{F_{\nu \alpha}^{L}+\frac{1}{2} U^{\dagger} F_{\nu \alpha}^{R} U, L_{\beta}\right\} .
\end{aligned}
$$

A physically irrelevant polynomial in the external fields $\ell, r$ has been omitted in the anomalous current (3.7).

Specializing to the dominant octet operator in $\mathcal{H}_{\text {eff }}^{\Delta S=1}[\mathrm{Eq} \cdot(2.1)]$,

$$
\begin{aligned}
Q_{-} & =Q_{2}-Q_{1} \\
Q_{1} & =\bar{s} \gamma^{\mu}\left(1-\gamma_{5}\right) d \bar{u} \gamma_{\mu}\left(1-\gamma_{5}\right) u \\
Q_{2} & =\bar{s} \gamma^{\mu}\left(1-\gamma_{5}\right) u \bar{u} \gamma_{\mu}\left(1-\gamma_{5}\right) d
\end{aligned}
$$

one obtains the following bosonized form of $O\left(p^{4}\right)$ in the factorizable approximation for the odd-parity part $[11,12]$ :

$$
\begin{aligned}
Q_{-} \text {(fact) } \leftrightarrow & \frac{F^{2}}{16 \pi^{2}}\left(2 i \varepsilon^{\mu \nu \alpha \beta}\left\langle\lambda L_{\mu}\right\rangle\left\langle L_{\nu} L_{\alpha} L_{\beta}\right\rangle\right. \\
& +\left\langle\lambda\left[U^{\dagger} \tilde{F}_{R}^{\mu \nu} U, L_{\mu} L_{\nu}\right]\right\rangle \\
& +3\left\langle\lambda L_{\mu}\right\rangle\left\langle\left(\tilde{F}_{L}^{\mu \nu}+U^{\dagger} \tilde{F}_{R}^{\mu \nu} U\right) L_{\nu}\right\rangle \\
& \left.+\left\langle\lambda L_{\mu}\right\rangle\left\langle\left(\tilde{F}_{L}^{\mu \nu}-U^{\dagger} \tilde{F}_{R}^{\mu \nu} U\right) L_{\nu}\right\rangle\right) .
\end{aligned}
$$

Comparison with the general weak Lagrangian $\mathcal{L}_{4}^{\Delta S=1}$ of $O\left(p^{4}\right)$ in (2.7), (2.8) shows that all the possible octet operators proportional to the $\varepsilon$ tensor $\left(W_{28}, W_{29}, W_{30}\right.$ and $\left.W_{31}\right)$ appear in $Q_{-}$(fact) in (3.9). Thus, in a slightly counter-intuitive way, the chiral anomaly contributes to all the coefficients $N_{28}, \ldots, N_{31}$ of normal octet operators. Moreover, the non-factorizable parts, which automatically have the right octet transformation property (they do not get any contribution from the anomaly), must be of the same form (3.9). The corresponding coefficients will differ from those in Eq. (3.9). In fact, they must depend on the QCD scale $\mu$ to cancel the $\mu$-dependence of the Wilson coefficients in the $\Delta S=1$ effective Hamiltonian [25]. 
Since all octet operators in $\mathcal{H}_{\text {eff }}^{\Delta S=1}$ produce the same structure (3.9), the $\Delta S=1$ effective Lagrangian in the anomalous parity sector of $O\left(p^{4}\right)$ can be characterized by the coefficients [11]

$$
\begin{aligned}
N_{28}^{\text {an }} & =\frac{a_{1}}{8 \pi^{2}} & N_{29}^{\text {an }} & =\frac{a_{2}}{32 \pi^{2}} \\
N_{30}^{\text {an }} & =\frac{3 a_{3}}{16 \pi^{2}} & N_{31}^{\text {an }} & =\frac{a_{4}}{16 \pi^{2}} .
\end{aligned}
$$

From the dominance of the octet operator $Q_{-}$, we expect the dimensionless coefficients $a_{i}$ to be positive and of order 1 . Unlike in the normal parity sector at $O\left(p^{2}\right)$ [25], the dominant penguin operator $Q_{6}$ does not contribute to the coefficients (3.10) in the factorizable approximation because there are no (pseudo-) scalar external fields in $S_{W Z W}$. Since $Q_{6}$ contributes constructively to the $O\left(p^{2}\right)$ weak coupling $G_{8}[25]$, which is pulled out in the definition of $\mathcal{L}_{4}^{\Delta S=1}$ in (2.7), we expect the $a_{i}$ to be actually smaller than one. The enhancement at $O\left(p^{4}\right)$ of the $\Delta I=1 / 2 K \rightarrow 2 \pi$ amplitudes [18] lends additional support to this expectation.

We are now in a position to determine all couplings relevant to nonleptonic $K$ decays to which the chiral anomaly contributes in a direct way via $\mathcal{L}_{4}^{\Delta S=1}$ in $(2.7),(2.8)$ with coefficients $(3.10)$. Restricting our attention to kinematically allowed $K$ decays ( $\leq 3$ pions, any number of photons), we obtain

$$
\begin{aligned}
& W_{28}= \frac{12 \sqrt{2} i}{F^{4}} \varepsilon_{\mu \nu \rho \sigma} \partial^{\mu} K^{0} \partial^{\nu} \pi^{0} D^{\rho} \pi^{+} D^{\sigma} \pi^{-}+\ldots \\
& W_{29}= \frac{4 i e}{F^{3}} \widetilde{F}_{\mu \nu}\left\{3 K^{+} \partial^{\mu} \pi^{0} D^{\nu} \pi^{-}+\sqrt{2} K^{0}\left(D^{\mu} \pi^{+} D^{\nu} \pi^{-}+\frac{i e}{2} F^{\mu \nu} \pi^{+} \pi^{-}\right)\right\} \\
&+\frac{e}{F^{4}} \widetilde{F}_{\mu \nu}\left\{6 K^{+} \pi^{0} D^{\mu} \pi^{-} \partial^{\nu} \pi^{0}-4 D^{\mu} K^{+} D^{\nu} \pi^{-} \pi^{+} \pi^{-}+4 K^{+} \pi^{-} D^{\mu} \pi^{+} D^{\nu} \pi^{-}\right. \\
&\left.+3 \sqrt{2} \partial^{\mu} K^{0} \partial^{\nu} \pi^{0} \pi^{+} \pi^{-}+\sqrt{2}\left(4 K^{0} \partial^{\mu} \pi^{0}-\partial^{\mu} K^{0} \pi^{0}\right)\left(\pi^{-} \stackrel{\leftrightarrow}{D^{\nu}} \pi^{+}\right)\right\}+\ldots \\
& W_{30}= \frac{4 i e}{F^{3}} \widetilde{F}_{\mu \nu} K^{+} D^{\mu} \pi^{-} \partial^{\nu} \pi^{0} \\
&+\frac{e}{F^{4}} \widetilde{F}_{\mu \nu}\left\{-2 K^{+} \pi^{0} D^{\mu} \pi^{-} \partial^{\nu} \pi^{0}-5 \sqrt{2} \partial^{\mu} K^{0} \partial^{\nu} \pi^{0} \pi^{+} \pi^{-}\right. \\
&\left.+\sqrt{2} K^{0} \partial^{\mu} \pi^{0}\left(\pi^{+} \stackrel{\leftrightarrow}{D}^{\nu} \pi^{-}\right)\right\}+\ldots \\
& \frac{2 \sqrt{2} i e}{F^{3}} \widetilde{F}_{\mu \nu} \partial^{\mu} K^{0}\left(\pi^{-} \stackrel{\leftrightarrow}{D^{\nu}} \pi^{+}\right) \\
& W_{31}=
\end{aligned}
$$


Table 1: A complete list of local anomalous non-leptonic weak $K$ decay amplitudes of $O\left(p^{4}\right)$ in the limit of CP conservation.

\begin{tabular}{|c|c|c|c|c|c|c|}
\hline Transition & $\mathcal{L}_{\mathrm{an}}^{\Delta S=1}$ & $W_{28}$ & $W_{29}$ & $W_{30}$ & $W_{31}$ & expt. \\
\hline$K^{+} \rightarrow \pi^{+} \pi^{0} \gamma$ & $\mathrm{X}$ & & $\mathrm{x}$ & $\mathrm{x}$ & & $\mathrm{x}$ \\
\hline$K^{+} \rightarrow \pi^{+} \pi^{0} \gamma \gamma$ & $\mathrm{x}$ & & $\mathrm{x}$ & $\mathrm{x}$ & & \\
\hline$K_{L} \rightarrow \pi^{+} \pi^{-} \gamma$ & & & $\mathrm{x}$ & & $\mathrm{x}$ & $\mathrm{x}$ \\
\hline$K_{L} \rightarrow \pi^{+} \pi^{-} \gamma \gamma$ & $\mathrm{x}$ & & $\mathrm{x}$ & & $\mathrm{x}$ & \\
\hline$K^{+} \rightarrow \pi^{+} \pi^{0} \pi^{0} \gamma$ & & & $\mathrm{x}$ & $\mathrm{X}$ & & $\mathrm{X}$ \\
\hline$K^{+} \rightarrow \pi^{+} \pi^{0} \pi^{0} \gamma \gamma$ & & & $\mathrm{X}$ & $\mathrm{x}$ & & \\
\hline$K^{+} \rightarrow \pi^{+} \pi^{+} \pi^{-} \gamma$ & & & $\mathrm{X}$ & & $\mathrm{X}$ & $\mathrm{x}$ \\
\hline$K^{+} \rightarrow \pi^{+} \pi^{+} \pi^{-} \gamma \gamma$ & & & $\mathrm{X}$ & & $\mathrm{X}$ & \\
\hline$K_{L} \rightarrow \pi^{+} \pi^{-} \pi^{0} \gamma$ & & $\mathrm{x}$ & $\mathrm{x}$ & $\mathrm{x}$ & & \\
\hline$K_{S} \rightarrow \pi^{+} \pi^{-} \pi^{0} \gamma(\gamma)$ & & & $\mathrm{x}$ & $\mathrm{x}$ & $\mathrm{x}$ & \\
\hline
\end{tabular}

We collect this information in Table 1 where all local contributions from either the Lagrangian $\mathcal{L}_{\text {an }}^{\Delta S=1}$ in (3.3) or the direct terms of $O\left(p^{4}\right)$ to all kinematically allowed non-leptonic $K$ decays are listed. A separate column indicates whether the corresponding decay has been observed experimentally. We emphasize that the transitions with either three pions and/or two photons in the final state are in general also subject to non-local reducible anomalous contributions of the type shown in Fig. ??.

Finally, we observe that in the non-leptonic weak sector the chiral anomaly contributes only to radiative $K$ decays.

\section{General features of $K \rightarrow \pi \pi \gamma$ decays}

The amplitude for $K(P) \rightarrow \pi_{1}\left(p_{1}\right)+\pi_{2}\left(p_{2}\right)+\gamma(q)$ is decomposed into an electric amplitude $E\left(x_{i}\right)$ and a magnetic amplitude $M\left(x_{i}\right)$ :

$$
A(K \rightarrow \pi \pi \gamma)=\varepsilon^{\mu}(q)^{*}\left[E\left(x_{i}\right)\left(p_{1} q p_{2 \mu}-p_{2} q p_{1 \mu}\right)+M\left(x_{i}\right) \varepsilon_{\mu \nu \rho \sigma} p_{1}^{\nu} p_{2}^{\rho} q^{\sigma}\right] / M_{K}^{3}
$$




$$
x_{i}=\frac{P p_{i}}{M_{K}^{2}} \quad(i=1,2), \quad x_{3}=\frac{P q}{M_{K}^{2}}, \quad x_{1}+x_{2}+x_{3}=1 .
$$

The invariant amplitudes $E\left(x_{i}\right), M\left(x_{i}\right)$ are dimensionless. Summing over the photon helicity, the differential decay distribution can be written as $\left(r_{i}=\right.$ $\left.M_{\pi_{i}} / M_{K}\right)$

$$
\begin{aligned}
\frac{\partial^{2} \Gamma}{\partial x_{1} \partial x_{2}}= & \frac{M_{K}}{4(4 \pi)^{3}}\left(\left|E\left(x_{i}\right)\right|^{2}+\left|M\left(x_{i}\right)\right|^{2}\right)\left[\left(1-2 x_{3}-r_{1}^{2}-r_{2}^{2}\right)\left(1-2 x_{1}+r_{1}^{2}-r_{2}^{2}\right) .\right. \\
& \left.\left(1-2 x_{2}+r_{2}^{2}-r_{1}^{2}\right)-r_{1}^{2}\left(1-2 x_{1}+r_{1}^{2}-r_{2}^{2}\right)^{2}-r_{2}^{2}\left(1-2 x_{2}+r_{2}^{2}-r_{1}^{2}\right)^{2}\right] .
\end{aligned}
$$

There is no interference between $E$ and $M$ as long as the photon helicity is not measured. In the following, we will not include the strong $\pi \pi$ rescattering phases in the amplitudes $E, M$ [27]. Of course, those phases should and are usually taken into account in the experimental analysis.

For most of the $K \rightarrow \pi \pi \gamma$ decays, the electric amplitude is dominated by the bremsstrahlung amplitude $E_{B}\left(x_{i}\right)$. This amplitude arises already at lowest $O\left(p^{2}\right)$ in CHPT. In fact, the following theorem $[10,17]$ shows that to $O\left(p^{2}\right)$ the $K \rightarrow \pi \pi \gamma$ amplitudes (actually $K \rightarrow \pi \pi(n \gamma)$ for any $n \geq 1$ ) are completely determined by $E_{B}\left(x_{i}\right)$. In other words, there is no additional information to $O\left(p^{2}\right)$ that would not already be contained in the corresponding non-radiative transitions $K \rightarrow \pi \pi$.

Theorem: Consider a general Lagrangian $\mathcal{L}_{2}\left(\varphi_{i}, D_{\mu} \varphi_{i}\right)(i=0,+,-)$ with at most two (electromagnetically gauge covariant) derivatives. In addition to the kinetic terms, there are only cubic interactions. Then the tree level amplitude for $\varphi_{0}, \varphi_{+}, \varphi_{-}$and any number $n$ of photons in the initial or final states factorizes,

$A\left(\varphi_{0} \varphi_{+} \varphi_{-} \gamma_{1} \ldots \gamma_{n}\right)=A_{B}\left(\varepsilon_{a}, q_{a}, p_{i}\right) A\left(\varphi_{0} \varphi_{+} \varphi_{-}\right) \quad a=1, \ldots, n \quad i=0,+,-$

$A\left(\varphi_{0} \varphi_{+} \varphi_{-}\right)$is the on-shell amplitude for the decay of either spin-0 particle into the other two and $A_{B}\left(\varepsilon_{a}, q_{a}, p_{i}\right)$ is the general bremsstrahlung amplitude independent of the structure of $\mathcal{L}_{2}$. 
The proof is straightforward and is relegated to Appendix A. Here, we add a few clarifying comments.

i. Although the notation is suggestive, the conclusion is not restricted to $O\left(p^{2}\right)$ in CHPT. Arbitrary mass terms in the chiral expansion fall under the general assumptions as long as there are at most two derivatives in the respective couplings. As a particular consequence, parts of the $O\left(p^{4}\right)$ CHPT corrections are covered by the theorem.

ii. The same constraints of gauge invariance and at most two derivatives imply that the amplitude $K \rightarrow \pi \gamma^{*} \ldots \gamma^{*}$ vanishes for any number of real or virtual photons [21]. There, only the gauge-invariant kinetic parts enter.

iii. A corresponding statement does not hold for more than three particles or more than two derivatives. We shall come back to this remark in Sect. 6. Note that this comment does not contradict Low's theorem [28], which is of course always valid.

iv. Although relatively trivial for $n=1$, the relation (4.3) can save a considerable amount of work for $n \geq 2$.

In the next section, we will try to estimate the dominant effects of $O\left(p^{6}\right)$ for the transitions $K_{L} \rightarrow \pi^{+} \pi^{-} \gamma$ and $K^{+} \rightarrow \pi^{+} \pi^{0} \gamma$ due to vector meson exchange. As is the case in general for non-leptonic weak transitions, there are two different mechanisms related to $\mathrm{V}$ exchange [29]. The first mechanism involves a strong VMD amplitude in connection with a non-leptonic weak transition on the external pseudoscalar meson legs. Given the strong amplitude, the weak VMD amplitude is unambiguously calculable through a weak rotation [21]. This is unfortunately not the case for the so-called direct weak terms corresponding to the weak Lagrangian $\mathcal{L}_{6}^{\Delta S=1}$ in the present situation. Even at $O\left(p^{4}\right)$, one must resort to models to obtain estimates of such terms related to $\mathrm{V}$ exchange [20].

In Ref. [10], the so-called weak deformation model (WDM) [29] was used to estimate the direct weak terms for the magnetic amplitudes of $O\left(p^{6}\right)$. Another model that has been used frequently in non-leptonic weak transitions (see [25] and references quoted therein) is the factorization model (FM). The 
FM is motivated by large- $N_{C}$ arguments ${ }^{1}$ and can be defined as (keeping only the octet part)

$$
\mathcal{L}_{\mathrm{FM}}=4 k_{f} G_{8}\left\langle\lambda \frac{\delta S}{\delta \ell_{\mu}} \frac{\delta S}{\delta \ell^{\mu}}\right\rangle+\text { h.c. }
$$

where $S$ is the CHPT action for the strong interactions and

$$
\frac{\delta S}{\delta \ell_{\mu}}=: J_{L}^{\mu}=J_{L, 1}^{\mu}+J_{L, 3}^{\mu}+J_{L, 5}^{\mu}+\ldots \quad J_{L, 1}^{\mu}=-\frac{i}{2} F^{2} U^{\dagger} D^{\mu} U
$$

is the corresponding left-chiral current. The constant $k_{f}$ is a fudge factor which the naive FM puts equal to one. ${ }^{2}$ As shown in Ref. [20], the WDM can be expressed through the Lagrangian

$$
\mathcal{L}_{\mathrm{WDM}}=2 G_{8}\left\langle\lambda\left\{J_{L, 1}^{\mu}, \frac{\delta S}{\delta \ell^{\mu}}\right\}\right\rangle+\text { h.c. }
$$

This Lagrangian formulation of the WDM immediately leads to the result [20] that $\mathcal{L}_{\text {WDM }}$ is a special case of $\mathcal{L}_{\mathrm{FM}}$ for $k_{f}=1 / 2$ to $O\left(p^{4}\right)$. However, starting at $O\left(p^{6}\right)$ the FM has additional terms not contained in the WDM.

For the transitions of interest here, we are only concerned with the magnetic amplitudes of $O\left(p^{6}\right)$. Since the strong action $S$ of $O\left(p^{4}\right)$ [8] has no terms with an $\varepsilon$ tensor except for the anomaly, the relevant FM Lagrangian consists of the following two terms:

$$
\mathcal{L}_{\mathrm{FM}}\left(M_{6}\right)=4 k_{f} G_{8}\left\{\left\langle\lambda\left\{\frac{\delta S_{2}}{\delta \ell_{\mu}}, \frac{\delta S_{6}}{\delta \ell^{\mu}}\right\}\right\rangle+\left\langle\lambda\left\{\frac{\delta S_{4}}{\delta \ell_{\mu}}, \frac{\delta S_{\mathrm{WZW}}}{\delta \ell^{\mu}}\right\}\right\rangle\right\}+\text { h.c. }
$$

Comparing with $\mathcal{L}_{\mathrm{WDM}}$ in $(4.6)$, the first term of $\mathcal{L}_{\mathrm{FM}}\left(M_{6}\right)$ reduces again to the WDM for $k_{f}=1 / 2$ since

$$
\frac{\delta S_{2}}{\delta \ell_{\mu}}=J_{L, 1}^{\mu}
$$

We can therefore simply multiply the WDM amplitudes derived in Ref. [10] by $2 k_{f}$ to get the corresponding FM amplitudes.

\footnotetext{
${ }^{1} \mathrm{~A}$ more systematic treatment of the large- $N_{C}$ expansion in this connection can be found in Ref. [30].

${ }^{2}$ For the weak anomalous action of Sect. $3, k_{f}=1$ corresponds to $a_{i}=1(i=1, \ldots, 4)$ in Eq. (3.10).
} 
The second term in Eq. (4.7) involves the anomalous current (3.7) and the normal current $\delta S_{4} / \delta \ell_{\mu}$ of $O\left(p^{3}\right)$. It is well-known [31] that the dominant terms in $S_{4}$ are due to spin-1 exchange. Taking the special form of the anomalous current in Eq. (3.7) into account and restricting ourselves to couplings sensitive to spin-1 exchange [31], one finds that a single term in $S_{4}$, albeit the one with the biggest coupling constant $L_{9}$, can contribute to $K \rightarrow \pi \pi \gamma$ decays via (4.7). Omitting all terms irrelevant for our transitions, the (matrix) current of $O\left(p^{3}\right)$ is given by

$$
\frac{\delta S_{4}}{\delta \ell^{\mu}}=i L_{9} \partial^{\nu}\left(\partial_{\nu} U^{\dagger} \partial_{\mu} U-\partial_{\mu} U^{\dagger} \partial_{\nu} U\right)+\ldots
$$

\section{$5 \quad$ Phenomenology of $K \rightarrow \pi \pi \gamma$ decays}

From the analysis of Sect. 3 summarized in Table 1, the chiral anomaly is seen to contribute only to the decays $K^{+} \rightarrow \pi^{+} \pi^{0} \gamma$ and $K_{L} \rightarrow \pi^{+} \pi^{-} \gamma$ at $O\left(p^{4}\right)$. In this section, we perform a detailed phenomenological analysis of these decays. For completeness, we include some remarks about the remaining $K \rightarrow \pi \pi \gamma$ decays referring to and commenting on recent work.

\section{$\mathbf{5 . 1} \quad K_{L, S} \rightarrow \pi^{0} \pi^{0} \gamma$}

For the decays $K^{0} \rightarrow \pi^{0} \pi^{0} \gamma$, Bose statistics implies

$$
\begin{aligned}
E\left(x_{2}, x_{1}\right) & =-E\left(x_{1}, x_{2}\right) \\
M\left(x_{2}, x_{1}\right) & =-M\left(x_{1}, x_{2}\right) .
\end{aligned}
$$

In the limit where $\mathrm{CP}$ is conserved, the amplitude for $K_{L}\left(K_{S}\right)$ is purely electric (magnetic).

The transition $K_{L} \rightarrow \pi^{0} \pi^{0} \gamma$ has recently been considered in the literature $[24,32]$. Eq. (5.1) implies the absence of a local amplitude of $O\left(p^{4}\right)$, or more generally the absence of an E1 amplitude. Although this by itself does not imply a vanishing one-loop amplitude (as can be seen in the case of $K_{L} \rightarrow$ $\pi^{+} \pi^{-} \gamma$ later in this section), Funck and Kambor [24] have shown that it does indeed vanish for a real photon. For a virtual photon, the one-loop 
amplitude is non-zero. In fact, it is divergent and it gets renormalized by the same combination of weak counterterms [24]

$$
2 N_{14}+N_{15}
$$

appearing in the transition $K_{1}^{0} \rightarrow \pi^{0} \gamma^{*}$ [33].

Thus, the decay $K_{L} \rightarrow \pi^{0} \pi^{0} \gamma$ with a real photon is at least $O\left(p^{6}\right)$ in CHPT. In fact, chiral symmetry permits local octet couplings of $O\left(p^{6}\right)$ contributing to this transition. A typical term, compatible with all symmetries, is provided by ${ }^{3}$

$$
\frac{1}{2}\left\langle\left\{\Delta, f_{+}^{\mu \nu}\right\}\left(\nabla_{\lambda} u_{\mu} u^{\lambda} u_{\nu}+u_{\nu} u^{\lambda} \nabla_{\lambda} u_{\mu}\right)\right\rangle .
$$

A survey of vector meson couplings of $O\left(p^{3}\right)$ [34] shows that there is no strong amplitude of $O\left(p^{6}\right)$ induced by $\mathrm{V}$ exchange that could contribute to $K_{L} \rightarrow \pi^{0} \pi^{0} \gamma$ via a weak rotation. It therefore seems legitimate to estimate the strength of the coupling (5.3) by naïve chiral dimensional analysis [35] as

$$
\mathcal{L}_{6}^{\Delta S=1}=\frac{G_{8}}{2(4 \pi)^{4}}\left\langle\left\{\Delta, f_{+}^{\mu \nu}\right\}\left(\nabla_{\lambda} u_{\mu} u^{\lambda} u_{\nu}+u_{\nu} u^{\lambda} \nabla_{\lambda} u_{\mu}\right)\right\rangle+\text { h.c. }+\ldots
$$

The corresponding amplitude for $K_{L} \rightarrow \pi^{0} \pi^{0} \gamma$ is

$$
E_{6}\left(x_{1}, x_{2}\right)=\frac{4 i G_{8} e M_{K}^{5}}{3(4 \pi)^{4} F^{3}}\left(x_{1}-x_{2}\right),
$$

yielding a branching ratio

$$
\left.B R\left(K_{L} \rightarrow \pi^{0} \pi^{0} \gamma\right)\right|_{O\left(p^{6}\right)}=7 \cdot 10^{-11}
$$

By relating $K_{L} \rightarrow \pi^{0} \pi^{0} \gamma$ to the decay $K_{L} \rightarrow \pi^{+} \pi^{-} \gamma$ (which is dominantly M1), Heiliger and Sehgal obtain a considerably bigger estimate [32] $\left.B R\left(K_{L} \rightarrow \pi^{0} \pi^{0} \gamma\right)\right|_{\mathrm{HS}}=1 \cdot 10^{-8}$ together with $\left.B R\left(K_{S} \rightarrow \pi^{0} \pi^{0} \gamma\right)\right|_{\mathrm{HS}}=$ $1.7 \cdot 10^{-11}$.

\footnotetext{
${ }^{3}$ For our purposes, the covariant derivatives in $(5.3),(5.4)$ can be replaced by normal ones.
} 


\section{$5.2 \quad K_{S} \rightarrow \pi^{+} \pi^{-} \gamma$}

In the limit of $\mathrm{CP}$ conservation, the amplitudes for $K_{S} \rightarrow \pi^{+} \pi^{-} \gamma$ obey the symmetry relations

$$
\begin{aligned}
E\left(x_{-}, x_{+}\right) & =E\left(x_{+}, x_{-}\right) \\
M\left(x_{-}, x_{+}\right) & =-M\left(x_{+}, x_{-}\right) .
\end{aligned}
$$

To $O\left(p^{4}\right)$, the amplitude is therefore purely electric. In addition to the bremsstrahlung amplitude of $O\left(p^{2}\right)$ (cf. theorem (4.3)), the loop and counterterm amplitudes of $O\left(p^{4}\right)$ have recently been calculated by D'Ambrosio, Miragliuolo and Sannino [36]. The local contribution of $O\left(p^{4}\right)$ is proportional to

$$
N_{14}-N_{15}-N_{16}-N_{17}
$$

and it is scale-independent $[19,20]$. The same combination of coupling constants appears in the electric amplitude for the decay $K^{+} \rightarrow \pi^{+} \pi^{0} \gamma[10]$. Consequently, the loop amplitudes for both $K_{S} \rightarrow \pi^{+} \pi^{-} \gamma$ and $K^{+} \rightarrow \pi^{+} \pi^{0} \gamma$ are finite.

At present, experimental data [37] are consistent with a pure bremsstrahlung amplitude. However, forthcoming facilities like DAPHNE [38] should be able to detect interference with the $O\left(p^{4}\right)$ amplitude that is expected to show up at the level of $10^{-6}$ in branching ratio (for $E_{\gamma}>20 \mathrm{MeV}$ ) $[36]$.

\section{$5.3 \quad K_{L} \rightarrow \pi^{+} \pi^{-} \gamma$}

The bremsstrahlung amplitude of $O\left(p^{2}\right)[10]$

$$
E_{B}\left(x_{i}\right)=\frac{\varepsilon e A\left(K_{1}^{0} \rightarrow \pi^{+} \pi^{-}\right)}{M_{K}\left(\frac{1}{2}-x_{+}\right)\left(\frac{1}{2}-x_{-}\right)} \quad p_{1}=p_{+}, \quad p_{2}=p_{-}
$$

violates $\mathrm{CP}$. Here $\varepsilon$ is the standard $\mathrm{CP}$ violation parameter in $K \rightarrow \pi \pi$ decays and we have neglected $\varepsilon^{\prime}$. From $O\left(p^{4}\right)$ on we assume CP conservation implying [cf. Eq. (5.7)]

$$
\begin{aligned}
E\left(x_{-}, x_{+}\right) & =-E\left(x_{+}, x_{-}\right) \\
M\left(x_{-}, x_{+}\right) & =M\left(x_{+}, x_{-}\right) .
\end{aligned}
$$


The dominant contribution of $O\left(p^{4}\right)$ occurs in the magnetic amplitude and it is due to the anomaly. As discussed in Sect. 3, there is no reducible anomalous amplitude of $O\left(p^{4}\right)$. The direct weak anomaly functional gives rise to $[11,12]$

$$
M_{4}=\frac{e G_{8} M_{K}^{3}}{2 \pi^{2} F}\left(a_{2}+2 a_{4}\right)
$$

in terms of the coupling constants $a_{i}$ defined in Eq. (3.10).

Because of (5.10) there is no local contribution to $E$ at $O\left(p^{4}\right)$. In contrast to $K_{L} \rightarrow \pi^{0} \pi^{0} \gamma$, there is however a finite one-loop amplitude. The relevant Feynman diagrams are shown in Fig. ??. The result of the loop calculation is proportional to the non-leptonic weak vertex occurring in Fig. ??, where the momenta of the corresponding three mesons are put on the mass shell. Consequently, only the diagrams of type $b$ give non-vanishing amplitudes for the $\pi^{ \pm} K_{1}^{0}$ and the $K^{ \pm} \eta$ intermediate states. In accordance with (5.10), the loop amplitude for $K_{L} \rightarrow \pi^{+} \pi^{-} \gamma$ takes the form [10]

$$
E_{4}^{\mathrm{loop}}\left(x_{+}, x_{-}\right)=\frac{i e G_{8} M_{K}\left(M_{K}^{2}-M_{\pi}^{2}\right)}{8 \pi^{2} F}\left[g\left(x_{-}\right)-g\left(x_{+}\right)\right],
$$

where the function $g(x)$ is defined in Appendix B. This result can now be compared with the bremsstrahlung amplitude (5.9):

$$
\left|\frac{E_{4}^{\text {loop }}}{E_{B}}\right|=\left|\frac{M_{K}^{2}}{\varepsilon(4 \pi F)^{2}}\left[g\left(x_{-}\right)-g\left(x_{+}\right)\right]\left(\frac{1}{2}-x_{+}\right)\left(\frac{1}{2}-x_{-}\right)\right| .
$$

Taking the maximum of this ratio over the whole Dalitz plot leads to the bounds

$$
\begin{aligned}
\left|\frac{E_{4}^{\mathrm{loop}}\left(\pi^{ \pm} K_{1}^{0}\right)}{E_{B}}\right| & \leq 1.1 \cdot 10^{-2}, \\
\left|\frac{E_{4}^{\mathrm{loop}}\left(K^{ \pm} \eta\right)}{E_{B}}\right| & \leq 0.1 \cdot 10^{-2}
\end{aligned}
$$

for the contributions of the $\pi^{ \pm} K_{1}^{0}$ and $K^{ \pm} \eta$ intermediate states, respectively. The smallness of the ratio of the two amplitudes is, of course, due to CP invariance, which is responsible for the antisymmetry in $x_{+}, x_{-}$of $E_{4}^{\text {loop }}$, forbidding in particular an electric dipole amplitude. Note also that because 
of $\arg \varepsilon \simeq \pi / 4$ there is only partial interference between $E_{4}^{\text {loop }}$ and $E_{B}$. It seems almost impossible to detect the loop amplitude.

For the electric amplitude $E$, the analysis to $O\left(p^{4}\right)$ is therefore more than sufficient. There are on the other hand strong experimental indications for the presence of a sizeable magnetic amplitude beyond $O\left(p^{4}\right)$. A recent analysis of $K_{L} \rightarrow \pi^{+} \pi^{-} \gamma$ at FNAL [14] confirms an earlier result from Brookhaven [13] finding evidence for a dependence of the direct emission amplitude on the photon energy. On the other hand, the dominant direct emission amplitude $M_{4}$ in (5.11) is a constant, independent of the photon energy.

At $O\left(p^{6}\right)$, CP invariance leads to the following most general form of the magnetic amplitude via Eq. (5.10):

$$
M_{6}\left(x_{+}, x_{-}\right)=a+b\left(x_{+}+x_{-}\right)=a+b-b x_{3}=\widehat{M}_{6}-b x_{3}, \quad x_{3}=\frac{P q}{M_{K}^{2}}=\frac{E_{\gamma}}{M_{K}},
$$

where $E_{\gamma}$ is the photon energy in the kaon rest frame. To $O\left(p^{6}\right)$, the total magnetic amplitude is therefore given by

$$
M\left(x_{+}, x_{-}\right)=M_{4}+M_{6}\left(x_{+}, x_{-}\right)=M_{4}+\widehat{M}_{6}-b x_{3}=\left(M_{4}+\widehat{M}_{6}\right)\left(1+c x_{3}\right) .
$$

From the distribution in $E_{\gamma}$ measured by E731 [14], one can extract [39] a value

$$
c=-1.7 \pm 0.5
$$

for the slope $c$, in agreement with the earlier measurement $[13,15]$.

How can CHPT account for this rather big slope? An early explanation was put forward by Lin and Valencia [40], who suggested a vector-mesondominated form factor in the $\pi^{+} \pi^{-}$invariant mass to be responsible for the slope. The experimental value (5.17) of the slope is in fact consistent with their model amplitude. Unfortunately, as already noted by Picciotto [41], the amplitude of Ref. [40] violates chiral symmetry. In the terminology of Sect. 3, their magnetic amplitude is of the reducible type, corresponding in particular to $a_{i}=0(i=1, \ldots, 4)$. To agree with our general result (5.11), their amplitude should therefore vanish at $O\left(p^{4}\right)$, which in fact it does not. The source of the problem seems to lie [41] in the model for combining the chiral anomaly and vector mesons.

Vector meson exchange contributes first at $O\left(p^{6}\right)$ to the amplitude $M$. This implies that the dependence on $E_{\gamma}$ due to a $V$ propagator is an effect 
of $O\left(p^{8}\right)$ and higher. Although not impossible, the big slope of Eq. (5.17) makes the interpretation as an $O\left(p^{8}\right)$ effect difficult to understand for the chiral practitioner. To make this feeling more quantitative, let us adopt the simplifying assumption that $M_{6}$ in (5.15) is entirely due to $V$ exchange. In this case

$$
M_{6}\left(x_{+}, x_{-}\right)=\frac{M_{6}^{0}}{1-\frac{\left(p_{+}+p_{-}\right)^{2}}{M_{V}^{2}}}=M_{6}^{0}\left[1+\frac{M_{K}^{2}}{M_{V}^{2}}\left(1-2 x_{3}\right)+\ldots\right] \quad\left(M_{V} \simeq M_{\rho}\right)
$$

and consequently

$$
\widehat{M}_{6}=M_{6}^{0}\left(1+\frac{M_{K}^{2}}{M_{V}^{2}}\right), \quad c=-\frac{2 M_{K}^{2} M_{6}^{0}}{M_{V}^{2}\left(M_{4}+\widehat{M}_{6}\right)} .
$$

Making the plausible assumption that the amplitude $\widehat{M}_{6}$ is at most equal to $M_{4}$ in magnitude, the observed sign of $c$ requires that $M_{4}$ and $\widehat{M}_{6}$ (or $M_{6}^{0}$ ) interfere constructively. Moreover, with $\left|M_{4}\right|>\left|\widehat{M}_{6}\right|$ the absolute value of the slope is bounded by

$$
|c|=\frac{2 M_{K}^{2} M_{6}^{0} / \widehat{M}_{6}}{M_{V}^{2}\left(1+M_{4} / \widehat{M}_{6}\right)}<\frac{M_{K}^{2}}{M_{V}^{2}+M_{K}^{2}}=0.3
$$

much too small to explain the measured value (5.17).

We are therefore led to interpret the slope $c$ as an effect of $O\left(p^{6}\right)$. Which are the dominant contributions of $O\left(p^{6}\right)$ ? First of all, there is a reducible amplitude due to the anomaly of the form

$$
\begin{gathered}
M_{6}^{\mathrm{anom}}=-\frac{e G_{8} M_{K}^{3}}{2 \pi^{2} F} F_{1} \\
F_{1}=\frac{1}{1-r_{\pi}^{2}}-\frac{(c-\sqrt{2} s)(c+2 \sqrt{2} \rho s)}{3\left(r_{\eta}^{2}-1\right)}+\frac{(\sqrt{2} c+s)(2 \sqrt{2} \rho c-s)}{3\left(r_{\eta^{\prime}}^{2}-1\right)} \\
r_{i}=M_{i} / M_{K}, \quad c=\cos \Theta, \quad s=\sin \Theta
\end{gathered}
$$

in the notation of Ref. [10]; $\Theta$ denotes the $\eta-\eta^{\prime}$ mixing angle and $\rho \neq 1$ takes into account possible deviations from nonet symmetry for the nonleptonic weak vertices (nonet symmetry is assumed for the strong WZW 
vertices $)$. At $O\left(p^{4}\right)\left(\Theta=0, M_{\eta^{\prime}} \rightarrow \infty\right), F_{1}$ vanishes because of the GellMann-Okubo mass formula. In the real world, the $\eta$ and $\eta^{\prime}$ contributions interfere destructively for $0 \leq \rho<1$ and $\Theta \simeq-20^{\circ}$ as in the similar case of the $K_{L} \rightarrow 2 \gamma$ amplitude. Although not really predictable with any precision, $F_{1}$ is dominated by the pion pole and certainly positive. We observe that $M_{4}$ (with $a_{i}>0$ ) and $M_{6}^{\text {anom }}$ interfere destructively, as already noted by Cheng [12], making a reliable estimate all the more difficult. At the present state of the art, the real challenge in $K_{L} \rightarrow \pi^{+} \pi^{-} \gamma$ is to understand the sign and magnitude of the slope $c$.

In the simplifying limit $M_{\pi}=0$, the strong VMD amplitude of $O\left(p^{6}\right)$ is unique. The relevant couplings are defined by the Lagrangian [34]

$$
\mathcal{L}_{V}=-\frac{i g_{V}}{2 \sqrt{2}}\left\langle\widehat{V}_{\mu \nu}\left[u^{\mu}, u^{\nu}\right]\right\rangle+h_{V}\left\langle\widehat{V}_{\mu}\left\{u_{\nu}, \widetilde{f}_{+}^{\mu \nu}\right\}\right\rangle+\ldots \quad \widehat{V}_{\mu \nu}=\nabla_{\mu} \widehat{V}_{\nu}-\nabla_{\nu} \widehat{V}_{\mu}
$$

for the vector meson resonance field $\widehat{V}_{\mu}$. Contracting the vector meson fields to produce an effective strong VMD Lagrangian of $O\left(p^{6}\right)$ proportional to $g_{V} h_{V}$ and applying a weak rotation leads to the weak VMD amplitude [10] $\left(M_{\pi}=0\right)$

$$
M_{6}^{\mathrm{VMD}}=2 C_{V}\left(1-3 x_{3}\right), \quad C_{V}=\frac{16 \sqrt{2} e G_{8} g_{V} h_{V} M_{K}^{5}}{3 M_{V}^{2} F} .
$$

To estimate the direct weak amplitude of $O\left(p^{6}\right)$ related to $V$ exchange, we make use of the FM as discussed in the previous section. With the Lagrangian defined in Eq. (4.7), one obtains $\left(M_{\pi}=0\right)$

$$
M_{6}^{\mathrm{FM}}=4 k_{f} C_{V} x_{3}+\frac{e G_{8} M_{K}^{5} L_{9}}{\pi^{2} F^{3}} k_{f}\left(2-5 x_{3}\right) .
$$

The first term reduces to the WDM amplitude for $k_{f}=1 / 2$ [10], whereas the second term has no analogue in the WDM and is proportional to the $O\left(p^{4}\right)$ coupling constant $L_{9}$ appearing in the current (4.9).

Altogether, we obtain for the magnetic amplitude

$$
M\left(x_{3}\right)=\frac{e G_{8} M_{K}^{3}}{2 \pi^{2} F}\left\{a_{2}+2 a_{4}-F_{1}+r_{V}\left[1+x_{3}\left(2 k_{f}-3\right)\right]+\frac{2 L_{9} M_{K}^{2}}{F^{2}} k_{f}\left(2-5 x_{3}\right)\right\}
$$




$$
r_{V}=\frac{64 \sqrt{2} \pi^{2} g_{V} h_{V} M_{K}^{2}}{3 M_{V}^{2}} \simeq 0.4 \simeq \frac{2 L_{9}^{r}\left(M_{\rho}\right) M_{K}^{2}}{F_{\pi}^{2}}
$$

with $[29,34,8]$

$$
g_{V} \simeq \frac{F_{\pi}}{\sqrt{2} M_{V}}, \quad\left|h_{V}\right| \simeq 3.7 \cdot 10^{-2}, \quad L_{9}^{r}\left(M_{\rho}\right) \simeq 6.9 \cdot 10^{-3}
$$

In contrast with more phenomenologically oriented treatments, CHPT as a quantum field theory permits a reliable determination of the relative signs of the various terms in the amplitude (5.25):

- Although we cannot predict a precise value for the quantity $a_{2}+2 a_{4}-F_{1}$, factorization discussed in Sect. $3\left(0<a_{i} \lesssim 1\right)$ strongly indicates a positive sign.

- Although the rates $\Gamma(V \rightarrow P \gamma)$ only determine $\left|h_{V}\right|$, the product $g_{V} h_{V}$, and therefore $r_{V}$, must be positive. The argument invokes yet another vector meson coupling constant $f_{V}[31,34]$. The product $f_{V} h_{V}$ governs the slope of the $\pi^{0}, \eta \rightarrow \gamma \gamma^{*} \rightarrow \gamma \ell^{+} \ell^{-}$amplitudes in the virtual photon mass [42]. Experimental evidence (see the discussion in Refs. [42, 43]) agrees with the predicted magnitude and fixes $f_{V} h_{V}>0$. On the other hand, $f_{V} g_{V} \simeq F_{\pi}^{2} / M_{V}^{2}[34]$ is known to be positive and so is therefore $g_{V} h_{V}$ (see also Ref.[44]).

- $L_{9}^{r}\left(M_{\rho}\right)$ is certainly positive [8]. In resonance approximation [31, 34] $L_{9}=\frac{1}{2} f_{V} g_{V}$, substantiating the previous argument.

Comparing the total magnetic amplitude (5.25) with the definition (5.16) of the slope parameter $c$, we infer that $c$ must be negative for all reasonable values of the factorization parameter $k_{f}\left(0<k_{f} \lesssim 1\right)$. To find out whether (5.25) can also explain the magnitude of the experimentally measured slope (5.17), we use the recent measurement [14]

$$
B R\left(E_{\gamma}>20 \mathrm{MeV}\right)_{\mathrm{DE}}=(3.19 \pm 0.16) \cdot 10^{-5}
$$

of the direct emission branching ratio to determine the quantity $a_{2}+2 a_{4}-F_{1}$ for given values of $k_{f}$. Then, the slope $c$ can be extracted from Eq. (5.25) both in magnitude and sign. 
Table 2: The slope parameter $c$ as a function of the factorization parameter $k_{f}$. The quantity $a_{2}+2 a_{4}-F_{1}$ is extracted from the measured branching ratio (5.27).

\begin{tabular}{|c|c|c|}
\hline$k_{f}$ & $a_{2}+2 a_{4}-F_{1}$ & $c$ \\
\hline 0 & 0.9 & -0.9 \\
0.5 & 0.6 & -1.3 \\
1 & 0.3 & -1.6 \\
\hline
\end{tabular}

The results are displayed in Table 2 for three representative values of $k_{f}$. The fitted values of $a_{2}+2 a_{4}-F_{1}$ document the expected strong destructive interference between the leading term $a_{2}+2 a_{4}$ and the $O\left(p^{6}\right)$ correction $F_{1}$. Our main results are the big values for $|c|$ as found experimentally. In view of Eq. (5.18), we may in addition expect an enhancement of $|c|$ by the propagator effect of $O\left(p^{8}\right)$. However, our analysis reinforces the previous conclusion that the slope parameter is dominantly an effect of $O\left(p^{6}\right)$. In summary, we cannot claim to be able to predict the rate for $K_{L} \rightarrow \pi^{+} \pi^{-} \gamma$, but CHPT establishes a correlation between the rate and the slope parameter $c$, in agreement with experiment.

In addition to the analysis of Ref. [40] already mentioned, several authors have addressed the decay $K_{L} \rightarrow \pi^{+} \pi^{-} \gamma$ recently. Cheng [12] used factorization for the $O\left(p^{4}\right)$ magnetic amplitude $\left(a_{2}=a_{4}=1\right)$. He has emphasized the need for a strong destructive interference between the leading contribution and higher-order terms like $F_{1}$, but he did not include $V$ exchange $\left(r_{V}=L_{9}=0\right.$ in $\left.(5.25)\right)$. In two more recent papers [45, 41], vector meson exchange is included. The magnetic amplitudes of Ko and Truong [45] and of Picciotto [41] agree to $O\left(p^{6}\right)$ with our amplitude $(5.25)$ in the (not very realistic) limit $a_{2}=a_{4}=0$ (no direct anomalous amplitude) and $k_{f}=0$ (pure VMD only). Within the hidden symmetry approach [46] for the "anomalous" couplings of vector mesons, they go beyond $O\left(p^{6}\right)$ by including in particular the vector meson propagators, but they find essentially no dependence of $M$ on the photon energy.

Further work on $K \rightarrow \pi \pi \gamma$ decays can be found in Ref. [47]. 


\section{$5.4 \quad K^{+} \rightarrow \pi^{+} \pi^{0} \gamma$}

The decay $K^{+} \rightarrow \pi^{+} \pi^{0} \gamma$ shares several features with $K_{L} \rightarrow \pi^{+} \pi^{-} \gamma$ :

- The bremsstrahlung amplitude is suppressed;

- The dominating contribution of $O\left(p^{4}\right)$ is due to the chiral anomaly;

- The one-loop amplitude is finite, but again very small.

The bremsstrahlung amplitude [10]

$$
E_{B}\left(x_{i}\right)=\frac{e A\left(K^{+} \rightarrow \pi^{+} \pi^{0}\right)}{M_{K} x_{3}\left(\frac{1}{2}-x_{0}\right)}, \quad p_{1}=p_{+}, \quad p_{2}=p_{0}
$$

includes the complete amplitude of $O\left(p^{2}\right)$ according to the theorem of Sect. 4 and it is suppressed by the $\Delta I=1 / 2$ rule.

The magnetic amplitude of $O\left(p^{4}\right)$ consists of both a reducible and a direct amplitude $[10,11]$ :

$$
M_{4}=\frac{e G_{8} M_{K}^{3}}{2 \pi^{2} F}\left(-1+\frac{3}{2} a_{2}-3 a_{3}\right) .
$$

Factorization suggests constructive interference between these two terms.

In contrast with $K_{L} \rightarrow \pi^{+} \pi^{-} \gamma$, there is now a local scale-independent contribution of $O\left(p^{4}\right)$ to the electric amplitude $E$ [10]:

$$
E_{4}^{\text {local }}=\frac{2 i e G_{8} M_{K}^{3}}{F}\left(N_{14}-N_{15}-N_{16}-N_{17}\right) .
$$

As already mentioned, the same combination of coupling constants appears in the amplitude for $K_{S} \rightarrow \pi^{+} \pi^{-} \gamma[36]$. By measuring the energy spectrum of the photon, the counterterm amplitude (5.30) can in principle be isolated through its interference with the bremsstrahlung amplitude (5.28). We can estimate the $\operatorname{size}^{4}$ of this interference by appealing to the FM which predicts $[20]$

$$
N_{14}-N_{15}-N_{16}-N_{17}=-k_{f} \frac{F_{\pi}^{2}}{2 M_{V}^{2}}=-7 \cdot 10^{-3} k_{f}
$$

\footnotetext{
${ }^{4}$ Although $N_{14}-N_{15}$ can be determined from the recent measurement of $K^{+} \rightarrow \pi^{+} e^{+} e^{-}$ $[48,33]$, the constants $N_{16}, N_{17}$ are still unknown.
} 
For $k_{f}>0$, the interference is predicted to be positive [10]:

$$
\frac{E_{4}^{\text {local }}}{E_{B}} \simeq 2.3 x_{3}\left(1-2 x_{0}\right)\left(-N_{14}+N_{15}+N_{16}+N_{17}\right) / 7 \cdot 10^{-3}
$$

The sign is well-determined because the ratio $G_{8} / G_{27}$ is known to be positive from $K \rightarrow 2 \pi$ decays (see Sect. 2). Except for small $E_{\gamma}\left(x_{3} \rightarrow 0,2 x_{0} \rightarrow 1\right)$ where bremsstrahlung is bound to dominate, the amplitude $E_{4}^{\text {local }}$ should be detectable. In fact, the experiment of Abrams et al. [49] is consistent with constructive interference between $E_{B}$ and $E_{4}^{\text {local }}$, but the available data [49] are not precise enough to separate the amplitudes $E-E_{B}$ and $M$ experimentally.

We now turn to the loop amplitude, which is necessarily finite. The Feynman diagrams are shown in Fig. ??. Similar to $K_{L} \rightarrow \pi^{+} \pi^{-} \gamma$, only the graphs of type $b$ with $\pi^{+} K_{1}^{0}$ and $K^{+} \eta$ intermediate states yield non-vanishing contributions in the octet limit $\left(G_{27}=0\right)$. The corresponding amplitude is given by

$$
E_{4}^{\mathrm{loop}}\left(x_{0}\right)=\frac{i e G_{8} M_{K}\left(M_{K}^{2}-M_{\pi}^{2}\right)}{8 \pi^{2} F} h\left(x_{0}\right)
$$

where the function $h(x)$ can again be found in Appendix B. The ratio of the loop amplitude to the bremsstrahlung amplitude can now be written in the form

$$
\left|\frac{E_{4}^{\mathrm{loop}}}{E_{B}}\right|=\left|\frac{M_{K}^{2} G_{8}}{24 \pi^{2} F^{2} G_{27}^{(3 / 2)}} x_{3}\left(\frac{1}{2}-x_{0}\right) h\left(x_{0}\right)\right|,
$$

which leads to the bounds

$$
\begin{aligned}
\left|\frac{E_{4}^{\mathrm{loop}}\left(\pi^{+} K_{1}^{0}\right)}{E_{B}}\right| & \leq 3.4 \cdot 10^{-2}, \\
\left|\frac{E_{4}^{\mathrm{loop}}\left(K^{+} \eta\right)}{E_{B}}\right| & \leq 0.7 \cdot 10^{-2} .
\end{aligned}
$$

At least in the foreseeable future, the loop amplitude can safely be neglected in comparison with the bremsstrahlung amplitude (5.28). On the other hand, the counterterm amplitude (5.30) should be within reach of facilities with intense $K^{+}$beams such as DAPHNE [38], not to speak of proper kaon factories. 
For $K_{L} \rightarrow \pi^{+} \pi^{-} \gamma$, it was essential to include $V$ exchange effects of $O\left(p^{6}\right)$, in particular to understand the slope parameter $c$. All the mechanisms discussed there also contribute to $K^{+} \rightarrow \pi^{+} \pi^{0} \gamma$. The weak VMD amplitude is $[10]$

$$
M_{6}^{\mathrm{VMD}}=-C_{V}
$$

and the direct weak amplitude is given by $\left(M_{\pi}=0\right)$

$$
M_{6}^{\mathrm{FM}}=2 k_{f} C_{V}+\frac{e G_{8} M_{K}^{5} L_{9}}{2 \pi^{2} F^{3}} k_{f}\left(3-8 x_{+}-2 x_{0}\right)
$$

in the framework of the FM. Altogether, we find for the total magnetic amplitude

$M=M_{4}+M_{6}=\frac{e G_{8} M_{K}^{3}}{4 \pi^{2} F}\left\{-2+3 a_{2}-6 a_{3}+r_{V}\left(2 k_{f}-1\right)+\frac{2 L_{9} M_{K}^{2}}{F^{2}} k_{f}\left(3-8 x_{+}-2 x_{0}\right)\right\}$.

Under the assumption that direct emission is entirely due to the magnetic part, experiments $[49,37]$ find a branching ratio

$$
B R\left(55<T_{\pi^{+}}(\mathrm{MeV})<90\right)=(1.8 \pm 0.4) \cdot 10^{-5}
$$

for the given cuts in the kinetic energy of the charged pion. Proceeding in a similar way as for $K_{L} \rightarrow \pi^{+} \pi^{-} \gamma$, we extract the quantity $A_{4}=-2+3 a_{2}-6 a_{3}$ from the measured rate. In order to exhibit the sensitivity to the $O\left(p^{6}\right)$ contributions, we first determine $A_{4}$ in the limit where $V$ exchange is turned off $\left(r_{V}=L_{9}=0\right)$ :

$$
A_{4}=-4.5 \pm 0.5 \text {. }
$$

For the physical values of $r_{V}$ and $L_{9}$ listed in Eq. (5.25), $A_{4}$ is found to be

$$
A_{4}=-4.1-0.3 k_{f} \pm 0.5, \quad 0 \leq k_{f} \leq 1 .
$$

We draw the following conclusions:

i. Compared with $K_{L} \rightarrow \pi^{+} \pi^{-} \gamma$, the $V$ exchange contributions are of less importance in the present case. Especially for $k_{f} \simeq 1$, the $O\left(p^{6}\right)$ terms are essentially negligible in the rate. 
ii. However, the last term in Eq. (5.38) shows a rather pronounced dependence on $x_{+}$. A high-precision analysis of the decay distribution in $T_{\pi+}$ may be able to reveal this dependence.

iii. The fitted values of $A_{4}$ are very much consistent with our expectations based on $a_{i} \lesssim 1$ (cf. Sect. 3).

iv. Because of the expected positive interference between $E_{B}$ and $E_{B}^{\text {local }}$, the coefficient $\left|A_{4}\right|$ is probably somewhat smaller than found above. Future experimental analysis should include an E1 amplitude of the type (5.30).

\section{Survey of the decays $K \rightarrow \pi \pi \gamma \gamma$ and $K \rightarrow$ $\pi \pi \pi \gamma(\gamma)$}

The complete list of non-leptonic $K$ decays with direct anomalous contributions can be found in Table 1 . In comparison with the dominant decays $K_{L} \rightarrow \pi^{+} \pi^{-} \gamma, K^{+} \rightarrow \pi^{+} \pi^{0} \gamma$ discussed in the previous section, the remaining processes are either suppressed by phase space or by the presence of an extra photon in the final state. It seems premature to perform a complete analysis of all those transitions to $O\left(p^{4}\right)$ in CHPT. Instead, we discuss their general features and illustrate the expected magnitude of anomalous contributions for two specific examples.

For the decays $K \rightarrow \pi \pi \gamma \gamma$, the general theorem of Sect. 4 applies. Thus, the $O\left(p^{2}\right)$ amplitude is completely given by bremsstrahlung. As for the $K \rightarrow \pi \pi \gamma$ transitions, direct anomalous amplitudes occur again only in the decays $K^{+} \rightarrow \pi^{+} \pi^{0} \gamma \gamma$ and $K_{L} \rightarrow \pi^{+} \pi^{-} \gamma \gamma$ where bremsstrahlung is suppressed. However, in both cases the dominant anomalous contributions are not the direct ones, but the rather trivial $\pi^{0} \rightarrow 2 \gamma$ transitions from $K \rightarrow 3 \pi$ intermediate states. ${ }^{5}$ Therefore, in order to isolate the non-trivial anomalous amplitudes in both $K^{+} \rightarrow \pi^{+} \pi^{0} \gamma \gamma$ and $K_{L} \rightarrow \pi^{+} \pi^{-} \gamma \gamma$ it is necessary to stay away from the pion pole in the $2 \gamma$-invariant mass. In practice, only the part of phase space with large $m_{2 \gamma}$ seems feasible for this purpose.

\footnotetext{
${ }^{5}$ Also $\eta \rightarrow 2 \gamma$ contributes, but to a much lesser extent.
} 
Let us consider the decay $K^{+}(P) \rightarrow \pi^{+}\left(p_{+}\right) \pi^{0}\left(p_{0}\right) \gamma\left(q_{1}\right) \gamma\left(q_{2}\right)$ as an example. As explained above, the amplitude at $O\left(p^{2}\right)$ is completely determined by $A\left(K^{+} \rightarrow \pi^{+} \pi^{0}\right)$, which is suppressed by the $\Delta I=1 / 2$ rule:

$$
\begin{aligned}
E^{(1)} \varepsilon^{\mu}\left(q_{1}\right)^{*} \varepsilon^{\nu}\left(q_{2}\right)^{*} . \\
\quad \cdot\left\{-\frac{1}{P q_{1}} \frac{1}{p_{+} q_{2}} P_{\mu} p_{+\nu}-\frac{1}{P q_{2}} \frac{1}{p_{+} q_{1}} p_{+\mu} P_{\nu}+\right. \\
\quad+\frac{1}{P\left(q_{1}+q_{2}\right)-q_{1} q_{2}}\left[\frac{1}{P q_{1}} P_{\mu}\left(P-q_{1}\right)_{\nu}+\frac{1}{P q_{2}}\left(P-q_{2}\right)_{\mu} P_{\nu}+g_{\mu \nu}\right] \\
\quad+\frac{1}{p_{+}\left(q_{1}+q_{2}\right)+q_{1} q_{2}}\left[\frac{1}{p_{+} q_{1}} p_{+\mu}\left(p_{+}+q_{1}\right)_{\nu}+\frac{1}{p_{+} q_{2}}\left(p_{+}+q_{2}\right)_{\mu} p_{+\nu}-g_{\mu \nu}(6,1,)\right.
\end{aligned}
$$

with $E^{(1)}=e^{2} A\left(K^{+} \rightarrow \pi^{+} \pi^{0}\right)$.

At $O\left(p^{4}\right)$, the electric amplitude is given by

$$
\begin{aligned}
& \varepsilon^{\mu}\left(q_{1}\right)^{*} \varepsilon^{\nu}\left(q_{2}\right)^{*} . \\
& \quad \cdot\left\{E ^ { ( 2 ) } \left[\frac{1}{P q_{1}} P_{\mu}\left(p_{+} q_{2} p_{0 \nu}-p_{0} q_{2} p_{+\nu}\right)+\frac{1}{P q_{2}}\left(p_{+} q_{1} p_{0 \mu}-p_{0} q_{1} p_{+\mu}\right) P_{\nu}\right.\right. \\
& \quad-\frac{1}{p_{+} q_{1}} p_{+\mu}\left(\left(p_{+}+q_{1}\right) q_{2} p_{0 \nu}-p_{0} q_{2}\left(p_{+}+q_{1}\right)_{\nu}\right) \\
& \quad-\frac{1}{p_{+} q_{2}}\left(\left(p_{+}+q_{2}\right) q_{1} p_{0 \mu}-p_{0} q_{1}\left(p_{+}+q_{2}\right)_{\mu}\right) p_{+\nu} \\
& \left.\left.\quad+\left(p_{0 \mu} q_{1 \nu}+q_{2 \mu} p_{0 \nu}-\left(q_{1}+q_{2}\right) p_{0} g_{\mu \nu}\right)\right]+E^{(3)}\left(q_{2 \mu} q_{1 \nu}-q_{1} q_{2} g_{\mu \nu}\right)\right\},
\end{aligned}
$$

where the coefficients $E^{(2)}$ and $E^{(3)}$ are scale-independent combinations of the coupling constants $N_{i}$ :

$$
\begin{aligned}
& E^{(2)}=\frac{8 \pi i \alpha G_{8}}{F}\left(N_{14}-N_{15}-N_{16}-N_{17}\right), \\
& E^{(3)}=\frac{32 \pi i \alpha G_{8}}{3 F}\left(N_{14}-N_{15}-2 N_{18}\right) .
\end{aligned}
$$

Consequently, the one-loop contributions to the decay amplitude must be finite. From the similarity with the case of $K^{+} \rightarrow \pi^{+} \pi^{0} \gamma$, we expect them to be small. 
The contributions from the chiral anomaly enter in the magnetic amplitude, which is given by

$$
\begin{aligned}
& \varepsilon^{\mu}\left(q_{1}\right)^{*} \varepsilon^{\nu}\left(q_{2}\right)^{*} \varepsilon^{\alpha \beta \gamma \delta} . \\
& \quad \cdot\left\{M ^ { ( 1 ) } \left[-g_{\mu \delta} p_{+\gamma} q_{1 \alpha} p_{0 \beta} \frac{P_{\nu}}{P q_{2}}-g_{\nu \delta} p_{+\gamma} q_{2 \alpha} p_{0 \beta} \frac{P_{\mu}}{P q_{1}}\right.\right. \\
& \left.\quad+g_{\mu \delta}\left(p_{+}+q_{2}\right)_{\gamma} q_{1 \alpha} p_{0 \beta} \frac{p_{+\nu}}{p_{+} q_{2}}+g_{\nu \delta}\left(p_{+}+q_{1}\right)_{\gamma} q_{2 \alpha} p_{0 \beta} \frac{p_{+\mu}}{p_{+} q_{1}}+g_{\mu \gamma} g_{\nu \delta}\left(q_{1}-q_{2}\right)_{\alpha} p_{0 \beta}\right] \\
& \left.\quad+\left(M^{(2)}+M^{(3)}+M^{(4)}\right) g_{\mu \gamma} g_{\nu \delta} q_{1 \alpha} q_{2 \beta}\right\},
\end{aligned}
$$

with

$$
\begin{aligned}
M^{(1)} & =\frac{2 \alpha G_{8}}{\pi F}\left(1-\frac{3}{2} a_{2}+3 a_{3}\right) \\
M^{(2)} & =\frac{4 \alpha G_{8}}{3 \pi F} \\
M^{(3)} & =\frac{\alpha G_{8}}{3 \pi F} \frac{6 P p_{+}-3 M_{K}^{2}-2 M_{\pi}^{2}+2\left(q_{1}+q_{2}\right)^{2}}{\left(q_{1}+q_{2}\right)^{2}-M_{\pi}^{2}} \\
M^{(4)} & =\frac{2 \alpha G_{8}}{\pi F} \frac{P\left(p_{+}-p_{0}\right)}{\left(q_{1}+q_{2}\right)^{2}-M_{\eta}^{2}} .
\end{aligned}
$$

The term with $M^{(1)}$ is determined by the magnetic amplitude of $K^{+} \rightarrow$ $\pi^{+} \pi^{0} \gamma$. The second term, proportional to $M^{(2)}$, is generated by the Lagrangian (3.3). Finally, the last two terms are coming from a $K^{+} \rightarrow \pi^{+} \pi^{0} \pi^{0}$ $\left(K^{+} \rightarrow \pi^{+} \pi^{0} \eta\right)$ intermediate state, followed by a subsequent transition $\pi^{0} \rightarrow \gamma \gamma(\eta \rightarrow \gamma \gamma)$.

As long as the photon helicities are not measured, there is no interference between electric and magnetic amplitudes. Our numerical results for the various contributions to the branching ratio with three different cuts in the $2 \gamma$ invariant mass $m_{2 \gamma}^{2}=\left(q_{1}+q_{2}\right)^{2}$ are displayed in Tables 3 and 4 . For the coupling constants $N_{i}$ occurring in (6.3) we have chosen the values suggested by the FM with $k_{f}=1$. In the quantity $M^{(1)}$ of $(6.5)$ we have used the naive factorization values $a_{2}=a_{3}=1$.

The remaining cases $K \rightarrow 3 \pi \gamma(\gamma)$ are in general dominated by the bremsstrahlung amplitudes entering with full strength. However, it is important to realize that the theorem of Sect. 4 is not applicable for those 
Table 3: Contributions to the branching ratio $B R\left(K^{+} \rightarrow \pi^{+} \pi^{0} \gamma \gamma\right)$ in units of $10^{-11}$ from the electric amplitudes. The indices $i, j=1,2,3$ refer to $E^{(1)}, E^{(2)}, E^{(3)}$ of $(6.1)$ and $(6.2)$.

\begin{tabular}{|c|c|c|c|}
\hline$i, j$ & $B R_{i j}\left(m_{2 \gamma}>170 \mathrm{MeV}\right)$ & $B R_{i j}\left(m_{2 \gamma}>180 \mathrm{MeV}\right)$ & $B R_{i j}\left(m_{2 \gamma}>190 \mathrm{MeV}\right)$ \\
\hline \hline 1,1 & 11.1 & 4.3 & 1.3 \\
\hline 2,2 & 0.7 & 0.3 & 0.1 \\
\hline 3,3 & 0.7 & 0.4 & 0.2 \\
\hline 1,2 & 5.4 & 2.3 & 0.7 \\
\hline 1,3 & 4.8 & 2.4 & 0.9 \\
\hline 2,3 & 1.3 & 0.7 & 0.3 \\
\hline \hline sum & 23.9 & 10.3 & 3.5 \\
\hline
\end{tabular}

Table 4: Contributions to the branching ratio $B R\left(K^{+} \rightarrow \pi^{+} \pi^{0} \gamma \gamma\right)$ in units of $10^{-11}$ from the magnetic amplitudes. The indices $i, j=1,2,3,4$ refer to $M^{(1)}, M^{(2)}, M^{(3)}, M^{(4)}$ of $(6.4)$.

\begin{tabular}{|c|c|c|c|}
\hline$i, j$ & $B R_{i j}\left(m_{2 \gamma}>170 \mathrm{MeV}\right)$ & $B R_{i j}\left(m_{2 \gamma}>180 \mathrm{MeV}\right)$ & $B R_{i j}\left(m_{2 \gamma}>190 \mathrm{MeV}\right)$ \\
\hline \hline 1,1 & 47.2 & 22.2 & 8.0 \\
\hline 2,2 & 0.9 & 0.5 & 0.2 \\
\hline 3,3 & 22.0 & 8.5 & 2.7 \\
\hline 4,4 & 0.0 & 0.0 & 0.0 \\
\hline 1,2 & 12.7 & 6.7 & 2.7 \\
\hline 1,3 & -61.7 & -26.9 & -9.2 \\
\hline 1,4 & -1.9 & -0.9 & -0.3 \\
\hline 2,3 & -4.4 & -2.1 & 0.0 \\
\hline 2,4 & -0.1 & -0.1 & 0.1 \\
\hline 3,4 & 0.7 & 0.3 & 3.4 \\
\hline \hline sum & 15.2 & 8.3 & \\
\hline
\end{tabular}


decays. In other words, already at $O\left(p^{2}\right)$ in CHPT there are in general additional contributions to the amplitudes that are not of the bremsstrahlung type. Those leading-order contributions are interesting in themselves and will be discussed in detail elsewhere. Here, we are interested in the sensitivity required to detect the presence of direct anomalous amplitudes governed by the weak coupling constants $N_{28}, \ldots, N_{31}$. We emphasize that there are in addition anomalous amplitudes of the reducible type that are not covered by the Lagrangian (3.3), but are of the type shown in Fig. ??. Since the WZW functional has no free parameters, those contributions are completely determined by the octet coupling $G_{8}$ in the $O\left(p^{2}\right)$ weak Lagrangian (2.2). An example, in addition to the $\pi^{0}(\eta) \rightarrow \gamma \gamma$ vertex relevant for two-photon decays, is a transition of the type $K \rightarrow \pi \pi \rightarrow \pi \pi \pi \gamma$ where the second step occurs via the WZW functional.

An interesting case with direct anomalous contributions is provided by $K_{L} \rightarrow \pi^{+} \pi^{-} \pi^{0} \gamma$. From the explicit form of the octet operators $W_{28}, \ldots, W_{31}$ in Eq. (3.11) and using (3.10), one finds the direct anomalous coupling

$$
\mathcal{L}_{\text {anom }}^{\text {direct }}\left(K_{L} \rightarrow \pi^{+} \pi^{-} \pi^{0} \gamma\right)=\frac{3 e G_{8}}{16 \pi^{2} F^{2}}\left(8 a_{1}+a_{2}-10 a_{3}\right) \widetilde{F}^{\mu \nu} \partial_{\mu} K_{2}^{0} \partial_{\nu} \pi^{0} \pi^{+} \pi^{-} .
$$

This Lagrangian has a few interesting features. First of all, the coefficients are potentially rather big if we recall $a_{i}=O(1)$ from the dominance of factorizable contributions (Sect. 3). However, with the naive factorization values $a_{i}=1$ there would be almost complete destructive interference in (6.6). Finally, the decay $K_{L} \rightarrow \pi^{+} \pi^{-} \pi^{0} \gamma$ is the only experimentally accessible process sensitive to the weak coupling constant $N_{28}$. Therefore, this decay affords in principle an interesting possibility to check the structure of direct anomalous terms.

Unfortunately, the available phase space is small. Ignoring all other contributions, in particular the dominant bremsstrahlung amplitude, the Lagrangian (6.6) would give rise to a branching ratio

$$
\left.B R\left(K_{L} \rightarrow \pi^{+} \pi^{-} \pi^{0} \gamma\right)\right|_{\text {direct }}=\left(8 a_{1}+a_{2}-10 a_{3}\right)^{2} \cdot 2 \cdot 10^{-10}
$$

Since not even the bremsstrahlung part has so far been observed experimentally, a test of the anomalous coupling (6.6) may have to wait for a while. 


\section{Summary}

Anomalies play a fundamental role in our understanding of modern particle physics. Gauge symmetries should be free of anomalies in order to allow a consistent quantization of the corresponding field theory. Global symmetries, however, can be broken at the quantum level. A well-known example is the chiral anomaly $[1,2]$, present in quantum field theories with chiral structure, such as the standard model. They do not constitute any obstruction for a proper quantization. Moreover, they have important implications for particle physics.

In the standard model, the chiral anomaly manifests itself most directly in the low-energy interactions of the pseudo-Goldstone bosons of spontaneously broken chiral symmetry. Since anomalies have a short-distance origin, their effect is completely calculable. The translation from the fundamental quarkgluon level to the effective chiral level (mesons) is unaffected by hadronization problems. The Wess-Zumino-Witten functional [9] encodes all low-energy manifestations of the chiral anomaly in strong interactions, in the presence of arbitrary external vector and axial-vector fields.

It is straightforward to work out the experimental consequences of the anomaly for electromagnetic and semileptonic weak processes. In addition to the classical test via the two-photon decays of the neutral pseudoscalars $\left(\pi^{0} \rightarrow \gamma \gamma, \eta \rightarrow \gamma \gamma, \eta^{\prime} \rightarrow \gamma \gamma\right)$ or the $\gamma 3 \pi$ and $\gamma \pi^{+} \pi^{-} \eta$ interactions [5], the manifestations of the non-Abelian chiral anomaly have mainly been investigated in semileptonic kaon decays $[3,4,50]$. Tau decays into three or more hadrons have also been pointed out $[51,52,53]$ to be sensitive to the anomaly, especially the decays $\tau \rightarrow \nu_{\tau} \eta+n \pi(n \geq 2)[51,52]$, which (for small hadronic invariant mass) only get contributions from the WZW term. Unfortunately, the presence of resonances at the high $Q^{2}$ values relevant for the $\tau$ decay spoils the possibility of making a clean quantitative test of the anomaly predictions.

In this paper we have presented a systematic investigation of the relevance of the chiral anomaly in non-leptonic weak transitions. Within the framework of CHPT, the manifestations of the anomaly appear first at $O\left(p^{4}\right)$. They can be grouped in two different classes of anomalous amplitudes: reducible and direct contributions. 
The reducible amplitudes arise from the contraction of meson lines between a weak $\Delta S=1$ vertex and the WZW functional (Fig. ??). The socalled pole contributions can be given in closed form [10] as a local Lagrangian (3.3) which contributes only to the decays $K^{+} \rightarrow \pi^{+} \pi^{0} \gamma, K^{+} \rightarrow \pi^{+} \pi^{0} \gamma \gamma$ and $K_{L} \rightarrow \pi^{+} \pi^{-} \gamma \gamma$. There are other reducible contributions which cannot be written in local form. In the octet limit, all reducible anomalous amplitudes of $O\left(p^{4}\right)$ can be predicted in terms of the coupling $G_{8}$.

The direct anomalous contributions arise from the contraction of the $W$ boson field between a strong Green function on one side and the WZW functional on the other side. Their computation is not straightforward, because of the presence of strongly interacting fields on both sides of the $W$. Using the operator product expansion to integrate out the heavy fields $(W, t, b$, $c$ ), one gets an effective Hamiltonian in terms of four-quark operators, which must be realized at the bosonic level in the presence of the anomaly. The factorizable contribution can be calculated in terms of bosonic currents. Due to the non-renormalization theorem of the chiral anomaly [26], there are no QCD corrections to the anomalous current, which is directly obtained from the WZW functional. Moreover, the non-factorizable piece does not get any contribution from the WZW functional. Therefore, the bosonized form of the direct anomalous amplitude can be fully predicted [11]. At $O\left(p^{4}\right)$, the anomaly turns out to contribute to all the possible octet operators proportional to the $\varepsilon$ tensor $\left(W_{28}, W_{29}, W_{30}\right.$ and $\left.W_{31}\right)$. In spite of its anomalous origin, this contribution is chiral-invariant. Unfortunately, the coefficients of these four operators get also non-factorizable contributions of non-anomalous origin, which cannot be computed in a model-independent way. Therefore, we can only parametrize the final result [Eq. (3.10)] in terms of dimensionless coefficients $a_{i}(i=1, \ldots, 4)$, which are expected to be positive and of order one.

A complete list of all kinematically allowed non-leptonic $K$ decays that get local contributions from the anomaly at $O\left(p^{4}\right)$ is given in Table 1. Only radiative $K$ decays are sensitive to the anomaly in the non-leptonic sector. The most frequent "anomalous" decays $K_{L} \rightarrow \pi^{+} \pi^{-} \gamma$ and $K^{+} \rightarrow \pi^{+} \pi^{0} \gamma$ share the remarkable feature that the normally dominant bremsstrahlung amplitude is strongly suppressed, making the experimental verification of the anomalous amplitude substantially easier. This suppression has different origins: $K^{+} \rightarrow \pi^{+} \pi^{0}$ proceeds through the small 27 -plet part of the non- 
leptonic weak interactions, whereas $K_{L} \rightarrow \pi^{+} \pi^{-}$is CP-violating.

For $K_{L} \rightarrow \pi^{+} \pi^{-} \gamma$, the direct emission rate is completely dominated by the magnetic amplitude. There is however a strong destructive interference between the $O\left(p^{4}\right)$ contribution (5.11) and the anomalous reducible amplitude (5.21), first appearing at $O\left(p^{6}\right)$. This $O\left(p^{6}\right)$ contribution stems from corrections to the Gell-Mann-Okubo mass formula and is very sensitive to the $\eta-\eta^{\prime}$ mixing angle and to nonet-symmetry-breaking effects. This makes a reliable estimate of the rate very difficult. Moreover, there is an important VMD contribution at $O\left(p^{6}\right)$, which generates a sizeable dependence of the magnetic amplitude on the photon energy. Although we cannot make absolute predictions for this decay, CHPT establishes a correlation between the rate and the energy slope in agreement with experiment.

For $K^{+} \rightarrow \pi^{+} \pi^{0} \gamma$, there is a potentially sizeable electric amplitude interfering with bremsstrahlung. This interference must be taken into account in the experimental analysis to extract the contribution of the anomaly to the rate. The VMD contribution to the magnetic amplitude is less important than in $K_{L} \rightarrow \pi^{+} \pi^{-} \gamma$. Nevertheless, it generates a rather pronounced dependence on the charged pion energy. Fitting our formulae to the measured direct-emission rate, one gets a value for the anomalous $O\left(p^{4}\right)$ magnetic amplitude (5.29), in good agreement with the factorization estimate.

The remaining non-leptonic $K$ decays with direct anomalous contributions (Table 1) are either suppressed by phase space or by the presence of an extra photon in the final state. For the decays $K \rightarrow \pi \pi \gamma \gamma$, direct anomalous amplitudes occur again only for $K_{L} \rightarrow \pi^{+} \pi^{-} \gamma \gamma$ and $K^{+} \rightarrow \pi^{+} \pi^{0} \gamma \gamma$, where bremsstrahlung is suppressed. However, in both cases the dominant anomalous contributions are the trivial $\pi^{0} \rightarrow 2 \gamma$ transitions from $K \rightarrow 3 \pi$ intermediate states. The decays $K \rightarrow 3 \pi \gamma(\gamma)$ are in general dominated by the bremsstrahlung amplitudes entering with full strength. An interesting case with direct anomalous contributions is provided by $K_{L} \rightarrow \pi^{+} \pi^{-} \pi^{0} \gamma$, which is the only experimentally accessible process sensitive to the weak coupling $N_{28}$.

Although not as straightforward as for electromagnetic and semileptonic weak processes, non-leptonic $K$ decays offer interesting possibilities for experimental tests of the chiral anomaly. 


\section{Acknowledgements}

We are grateful to Erik Ramberg for information on the results of Ref. [14]. 


\section{Appendix A: Bremsstrahlung amplitudes for $K \rightarrow \pi \pi \gamma \ldots \gamma$}

The tree level generating functional $Z_{\text {tree }}[j, A]$ for connected $(3+n)$-point functions with three external spin- 0 legs and $n$ external photons can be written as

$$
Z_{\text {tree }}[j, A]=\int d^{4} x \mathcal{L}_{2}^{\mathrm{cubic}}\left(\varphi_{k}^{\mathrm{cl}}[j, A], D_{\mu} \varphi_{k}^{\mathrm{cl}}[j, A]\right) .
$$

$\mathcal{L}_{2}^{\text {cubic }}$ is the cubic part of the general Lagrangian $\mathcal{L}_{2}\left(\varphi_{k}, D_{\mu} \varphi_{k}\right)$ in the theorem of Sect. 4. The classical fields $\varphi_{k}[j, A]$ are solutions of the free equations of motion

$$
\left(D^{2}+M_{k}^{2}\right) \varphi_{k}^{\mathrm{cl}}=j_{k}, \quad D_{\mu} \varphi_{k}=\left(\partial_{\mu}-i e q_{k} A_{\mu}\right) \varphi_{k}, \quad k=+,-, 0
$$

with external sources $j_{k}$ in the presence of an external electromagnetic field $A_{\mu}$.

Using partial integration in the action, the most general gauge-invariant cubic interaction Lagrangian with at most two derivatives has the form

$$
\mathcal{L}_{2}^{\text {cubic }}=\varphi_{0}\left(a_{1} D^{2} \varphi_{+} \varphi_{-}+a_{2} \varphi_{+} D^{2} \varphi_{-}+a_{3} D_{\mu} \varphi_{+} D^{\mu} \varphi_{-}\right)+b \varphi_{0} \varphi_{+} \varphi_{-},
$$

where $a_{1}, a_{2}, a_{3}, b$ are coupling constants that may depend on the masses $M_{k}$. We use partial integration once more,

$$
\varphi_{0} D_{\mu} \varphi_{+} D^{\mu} \varphi_{-} \bumpeq \frac{1}{2}\left(D^{2} \varphi_{0} \varphi_{+} \varphi_{-}-\varphi_{0} D^{2} \varphi_{+} \varphi_{-}-\varphi_{0} \varphi_{+} D^{2} \varphi_{-}\right)
$$

to bring $\mathcal{L}_{2}^{\text {cubic }}$ into the final form

$$
\mathcal{L}_{2}^{\text {cubic }}=\varphi_{0}\left(a_{1}^{\prime} D^{2} \varphi_{+} \varphi_{-}+a_{2}^{\prime} \varphi_{+} D^{2} \varphi_{-}\right)+a_{3}^{\prime} D^{2} \varphi_{0} \varphi_{+} \varphi_{-}+b \varphi_{0} \varphi_{+} \varphi_{-} .
$$

In the generating functional $Z_{\text {tree }}$ in (A.1), the derivatives appear therefore only in the form of covariant d'Alembertians acting on the classical fields $\varphi_{k}^{\mathrm{cl}}$. Using the equations of motion (A.2), we may write

$$
D^{2} \varphi_{k}^{\mathrm{cl}}=D^{2}\left(D^{2}+M_{k}^{2}\right)^{-1} j_{k}=j_{k}-M_{k}^{2}\left(D^{2}+M_{k}^{2}\right)^{-1} j_{k} .
$$

Since the first term $j_{k}$ on the right-hand side does not contribute to on-shell amplitudes (amputated Green functions), we may replace $D^{2} \varphi_{k}^{\mathrm{cl}}$ by $-M_{k}^{2} \varphi_{k}^{\mathrm{cl}}$ 
everywhere in $\mathcal{L}_{2}^{\text {cubic }}\left(\varphi_{k}^{\text {cl }}, D_{\mu} \varphi_{k}^{\text {cl }}\right)$. Thus, the Lagrangian is equivalent to the non-derivative cubic Lagrangian

$$
\mathcal{L}_{2}^{\text {cubic }} \widehat{=} \varphi_{0} \varphi_{+} \varphi_{-}\left(-a_{1}^{\prime} M_{+}^{2}-a_{2}^{\prime} M_{-}^{2}-a_{3}^{\prime} M_{0}^{2}+b\right)
$$

for which the theorem is trivially satisfied. 


\section{Appendix B: Loop functions}

The kinematical functions $g(x)$ and $h(x)$ originate from the loop integral

$$
\int \frac{d^{4} k}{(2 \pi)^{4}} \frac{k^{\mu} k^{\nu}}{\left(k^{2}-M_{1}^{2}\right)\left((k+q)^{2}-M_{1}^{2}\right)\left((k-p)^{2}-M_{2}^{2}\right)}=i g^{\mu \nu} C_{20}\left(p^{2},(p+q)^{2}, M_{1}^{2}, M_{2}^{2}\right)+\ldots
$$

where $q^{2}=0$. The dots in (B.1) refer to terms that are irrelevant in our case. In the next step we define the (finite) function

$$
\bar{C}_{20}\left(p^{2},(p+q)^{2}, M_{1}^{2}, M_{2}^{2}\right)=C_{20}\left(p^{2},(p+q)^{2}, M_{1}^{2}, M_{2}^{2}\right)-C_{20}\left(p^{2}, p^{2}, M_{1}^{2}, M_{2}^{2}\right) .
$$

With this definition, the function $g(x)$ [used in (5.12)] and the function $h(x)$ of (5.33) are given by

$$
\begin{aligned}
& g(x)=(4 \pi)^{2} \frac{M_{K}^{2}}{p q}\left[\bar{C}_{20}\left(p^{2},(p+q)^{2}, M_{\pi}^{2}, M_{K}^{2}\right)+\bar{C}_{20}\left(p^{2},(p+q)^{2}, M_{K}^{2}, M_{\eta}^{2}\right)\right], \\
& h(x)=(4 \pi)^{2} \frac{M_{K}^{2}}{p q}\left[\bar{C}_{20}\left(p^{2},(p+q)^{2}, M_{\pi}^{2}, M_{K}^{2}\right)+\frac{2}{3} \bar{C}_{20}\left(p^{2},(p+q)^{2}, M_{K}^{2}, M_{\eta}^{2} \gamma \beta, 3\right)\right.
\end{aligned}
$$

where $p^{2}=M_{\pi}^{2}$ and $p q=M_{K}^{2}\left(\frac{1}{2}-x\right)$. 


\section{References}

[1] S.L. Adler, Phys. Rev. 177 (1969) 2426;

J.S. Bell and R. Jackiw, Nuovo Cim. 60A (1969) 47.

[2] W.A. Bardeen, Phys. Rev. 184 (1969) 1848.

[3] J. Bijnens, G. Ecker and J. Gasser, Semileptonic kaon decays, in The DAPHNE Physics Handbook, Eds. L. Maiani, G. Pancheri and N. Paver, INFN-Frascati (1992).

[4] G. Ecker, $K$ decays and the chiral anomaly, in Proc. of the 27th Rencontre de Moriond, Electroweak Interactions and Unified Theories, Ed. J. Tran Thanh Van, Eds. Frontières (Gif-sur-Yvette, 1992).

[5] J. Bijnens, Chiral perturbation theory and anomalous processes, to appear in Int. J. Mod. Phys. A.

[6] S. Weinberg, Physica 96A (1979) 327.

[7] J. Gasser and H. Leutwyler, Ann. Phys. 158 (1984) 142.

[8] J. Gasser and H. Leutwyler, Nucl. Phys. B250 (1985) 465.

[9] J. Wess and B. Zumino, Phys. Lett. 37B (1971) 95;

E. Witten, Nucl. Phys. B223 (1983) 422.

[10] G. Ecker, H. Neufeld and A. Pich, Phys. Lett. B278 (1992) 337.

[11] J. Bijnens, G. Ecker and A. Pich, Phys. Lett. B286 (1992) 341.

[12] H.-Y. Cheng, Phys. Rev. D42 (1990) 72.

[13] A.S. Carroll et al., Phys. Lett. 96B (1980) 407.

[14] E.J. Ramberg et al. (E731-FNAL), Phys. Rev. Lett. 70 (1993) 2525.

[15] L. Littenberg and G. Valencia, Rare and radiative kaon decays, FERMILAB-PUB-93/004-T, to appear in Annu. Rev. Nucl. Part. Sci., vol. 43 .

[16] F.J. Gilman and M.B. Wise, Phys. Rev. D20 (1979) 2392. 
[17] E. de Rafael, Nucl. Phys. B (Proc. Suppl.) 7A (1989) 1.

[18] J. Kambor, J. Missimer and D. Wyler, Phys. Lett. B261 (1991) 496.

[19] J. Kambor, J. Missimer and D. Wyler, Nucl. Phys. B346 (1990) 17.

[20] G. Ecker, J. Kambor and D. Wyler, Nucl. Phys. B394 (1993) 101.

[21] G. Ecker, A. Pich and E. de Rafael, Nucl. Phys. B303 (1988) 665.

[22] G. Ecker, A. Pich and E. de Rafael, Phys. Lett. B189 (1987) 363.

[23] H. Dykstra, J.M. Flynn and L. Randall, Phys. Lett. B270 (1991) 45.

[24] R. Funck and J. Kambor, Nucl. Phys. B396 (1993) 53.

[25] A. Pich and E. de Rafael, Nucl. Phys. B358 (1991) 311.

[26] S.L. Adler and W.A. Bardeen, Phys. Rev. 182 (1969) 1517.

[27] G. Costa and P.K. Kabir, Nuovo Cim. 51A (1967) 564.

[28] F.E. Low, Phys. Rev. 110 (1958) 974.

[29] G. Ecker, A. Pich and E. de Rafael, Phys. Lett. B237 (1990) 481.

[30] C. Bruno and J. Prades, Z. Phys. C57 (1993) 585.

[31] G. Ecker, J. Gasser, A. Pich and E. de Rafael, Nucl. Phys. B321 (1989) 311.

[32] P. Heiliger and L.M. Sehgal, Phys. Lett. B307 (1993) 182.

[33] G. Ecker, A. Pich and E. de Rafael, Nucl. Phys. B291 (1987) 692.

[34] G. Ecker, J. Gasser, H. Leutwyler, A. Pich and E. de Rafael, Phys. Lett. B223 (1989) 425.

[35] A. Manohar and H. Georgi, Nucl. Phys. B234 (1984) 189.

[36] G. D'Ambrosio, M. Miragliuolo and F. Sannino, $K_{S} \rightarrow \pi^{+} \pi^{-} \gamma$ : a laboratory for meson dynamics, Naples preprint INFNNA-IV-93/3. 
[37] Review of Particle Properties, Phys. Rev. D45 (1992) Part II.

[38] The DAPHNE Physics Handbook, Eds. L. Maiani, G. Pancheri and N. Paver, INFN-Frascati (1992).

[39] E.J. Ramberg, private communication.

[40] Y.C.R. Lin and G. Valencia, Phys. Rev. D37 (1988) 143.

[41] C. Picciotto, Phys. Rev. D45 (1992) 1569.

[42] G. Ecker, Chiral realization of the non-leptonic weak interactions, in Proc. 24th Int. Symposium on the Theory of Elementary Particles, Gosen (Berlin), Ed. G. Weigt (Zeuthen, 1991).

[43] J. Bijnens, A. Bramon and F. Cornet, Z. Phys. C46 (1990) 599.

[44] J. Prades, Massive spin-1 field chiral Lagrangian from an extended Nambu-Jona-Lasinio model of QCD, Marseille preprint CPT-93/P.2871.

[45] P. Ko and T.N. Truong, Phys. Rev. D43 (1991) R4.

[46] M. Bando, T. Kugo and K. Yamawaki, Phys. Reports 164 (1988) 115.

[47] A.A. Bel'kov, Yu.L. Kalinowskii, V.N. Pervushin and N.A. Sarikov, Yad. Fiz. 44 (1986) 690 [Sov. J. Nucl. Phys. 44 (1986) 448];

M. McGuigan and A.I. Sanda, Phys. Rev. D36 (1987) 1413;

S. Fajfer, Z. Phys. C45 (1989) 293;

M.K. Volkov and A.A. Osipov, JINR Rapid Communications 3 (1991) 67 ;

L.M. Sehgal and M. Wanninger, Phys. Rev. D46 (1992) 1035; E-ibid. D46 (1992) 5209;

H.-Y. Cheng, Direct CP violation in $K_{L} \rightarrow \pi^{+} \pi^{-} \gamma$, Stony Brook preprint ITP-SB-93-32;

A.A. Bel'kov, A.V. Lanyov and A. Schaale, Radiative kaon decays and CP violation, DESY preprint DESY 93-060.

[48] C. Alliegro et al., Phys. Rev. Lett. 68 (1992) 278.

[49] R.J. Abrams et al., Phys. Rev. Lett. 29 (1972) 1118;

V.N. Bolotov et al., Sov. J. Nucl. Phys. 45 (1987) 1023. 
[50] G. Kramer, W.F. Palmer and S.S. Pinsky, Phys. Rev. D30 (1984) 89; S.-C. Chao et al., Phys. Rev. D30 (1984) 1916; G. Kramer and W.F. Palmer, Phys. Rev. D36 (1987) 154.

[51] G. Kramer and W.F. Palmer, Z. Phys. C25 (1984) 195; ibid C39 (1988) 423 ;

E. Braaten, R.J. Oakes and S.-M. Tse, Phys. Rev. D36 (1987) 2188.

[52] A. Pich, Phys. Lett. B196 (1987) 561.

[53] J.J. Gómez-Cadenas, M.C. González-García and A. Pich, Phys. Rev. D42 (1990) 3093. 